\title{
Employer Strategies for Preventing Mental Health Related Work Disability: A Scoping Review'
}

I Ulrik Gensby2

PhD, Senior Scientist at Team Arbejdsliv ApS, Copenhagen, Denmark

I Hans Jørgen Limborg

PhD, Scientific Director at Team Arbejdsliv Aps, Copenhagen, Denmark

\section{Quenby Mahood}

MI, Manager of Library Services at Institute for Work and Health, Toronto, Canada

\section{Christian Ståhl}

PhD, Associate Professor at Department of Medical and Health Services, Helix Competence Centre, Linkoping University, Sweden

\section{Karen Albertsen}

PhD, Senior Scientist at Team Arbejdsliv Life Aps, Copenhagen, Denmark

\begin{abstract}
This study examined Nordic research on psychosocial work environment and disability management, specifically employer strategies for preventing work disability in common mental disorders (CMDs). A scoping review was performed to identify strategies across several research databases, alongside contact with content experts, hand-searching of non-indexed journals, and internet searches. Identification and selection of relevant studies, charting of data, and collating and summarizing of results was done using a six-step framework for conducting scoping reviews. Several key elements and knowledge gaps were identified in current prevention approaches and workplace initiatives across the included studies. We propose a program theory for workplace prevention of CMD-related work disability. The program theory may help specify employer strategies, and bridge activities with stakeholders outside the workplace.
\end{abstract}

\section{KEYWORDS}

Scoping review / mental health / work disability / employer / workplace / return to work

\section{Background and relevance of the problem}

ordic working life research has a long tradition for critical studies on work disability prevention that provides a consistent knowledge base on the central role of employers in developing and maintaining a healthy workforce (Hvid 2011; Kamp

\footnotetext{
${ }^{1}$ You can find this text and its DOI at https://tidsskrift.dk/njwls/index.

${ }^{2}$ Corresponding author: Ulrik Gensby, Team Arbejdsliv ApS, Høffdingsvej 22, 1 sal, 2500 Valby, Denmark. E-mail: ugge@teamarbejdsliv.dk.
} 
\& Nielsen 2013). Importantly, employers have responsibility to structure preventive initiatives, and their efforts to organize and manage human resources in working environments influence employee sickness absence understood as temporary or periodic inability to work (Geisen \& Harder 2011; Loisel \& Anema 2013). One of the major challenges for employers striving to prevent work disability is the magnitude of common mental disorders (CMDs) such as anxiety, depression, and stress-related illness (Andersen 2012; Corbiere 2009; McDowell \& Fossey 2015). From a cross-national perspective, musculoskeletal disorders no longer comprise the largest diagnostic group. Instead, CMDs are increasing among the working-age population, with depression and stress-related illness rising as the dominant public health problem among the Nordic countries (Prins 2013). The economic and social costs associated with high levels of CMD make a clear 'business case' for work disability prevention as a renewable area for employers to boost preventive legitimacy and approaches. Many employers do strive to establish a comprehensive preventive approach, while struggling to understand the complex ways in which CMDs are embedded within productivity, work organization, and social relations at work (MacEachen 2006). Facilitating new employer opportunities to prevent CMDrelated work disability has therefore been subject to much scientific and political debate, including a wide spectrum of research fields (Gallie 2011; Pomaki 2012; WHO 2006).

\section{Employer activation in work disability policy}

Several Nordic research studies have debated the employer role in work disability policies, and to what extent employers should be responsible to accommodate workers. Dealing with employer responsibility in organizing return to work (RTW), however, is a complex issue for several reasons. Employers do not receive a great deal of training in managing the process of RTW (Ekberg 2016; Stochkendahl 2015). Employers need capacity building in workplace strategies and how to coordinate separate activities to make them work together (Kärrholm 2007; Tjulin 2010). Contemporary sickness benefit policies and legal requirements do not always adequately prompt employers to engage in RTW, and often little guidance is given on specific requirements for work accommodation and follow-up outside the workplace (Clayton 2011; Seing 2015a). In contrast, recent administrative reforms seem to have been introducing continuous change in work activation and sickness benefit schemes with added responsibility for RTW coordination put on local welfare services (Aust 2015; Seing 2012). This makes it difficult to integrate inclusive working life with work activation policies (Andreassen \& Spjelkavik 2013). While recent reforms are debateable and manifested differently across the Nordic countries, the reforms have proven less likely to facilitate and maintain a strong workplace approach, and rather created a 'missing link' to employers in post injury/illness prevention (Clayton 2011; Ståhl 2009). Importanly, Nordic welfare states risk to miss critical employer resources that could serve a more systematic approach to bridge preventive activities and renew the role of employer as a stakeholder in social policy (Midtsundstad 2008). Interestingly, differences in work disability outcomes seem to be highly correlated with differences in applied workplace interventions across countries. The main implication of such research findings is that a policy change is needed to encourage more attention to employer efforts and workplace initiatives, through collaborative action at the workplace level (Anema 2009).

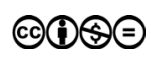




\section{The value of integrated prevention approaches}

Many practical and economic reasons exist to why the workplace may be the ideal setting for establishing and implementing initiatives to prevent CMD-related work disability. Mykletun and Harvey (2012) argue how 'the prospect of using the workplace to help prevent mental illness brings together the two opposing paradigms in occupational mental health'. They explain how the focus of any workplace initiative to prevent CMD should be on revealing the harmful components of the work environment with the intention of removing the exposure or buffering its effect when viewed from the 'toxic workstress' paradigm. Adopting the 'work-is-good-for-your-health' paradigm, workplace initiatives, such as modified work and partial sickness absence, should be considered as preventive initiatives in disability management in their own right (Mykletun \& Harvey 2012). Traditionally, scientists have been separating workplace interventions to prevent CMD-related work disability in primary, secondary, and tertiary prevention approaches (Murphy \& Sauter 2004). While there is a rationale for discussing and distinguishing prevention activities across prevention approaches, there are considerable limitations in implementing primary and secondary prevention activities as separated processes (Kristensen 2008). In the context of CMD-related work disability, preventive efforts may be initiated from a psychosocial work environment approach to prevent sickness absence, and/or a disability management approach to coordinate resources and promote RTW. Here, employers face challenges supporting the RTW process, while at the same time assessing and learning from issues in the working environment that could potentially 'trigger' the CMD condition, when implementing workplace initiatives and work practice change (Hasle 2009; Tjulin 2015). While considering the challenges of work-related stress, an integrated prevention approach remains an important subject for ongoing scientific refinement, particularly as a conceptual means to bridge preventive paradigms and to avoid thinking in silos (Harenstam 2005).

\section{Synthesizing research to inform and guide policy and practice}

The state of the evidence is in a constant change, and today's preventive methods do not necessarily contribute to the best preventive solutions of tomorrow's problems. One of the main drivers for innovative solutions is scientific research and knowledge exchange processes with key stakeholders, which can offer practitioners and policy makers a new line of reference to substantiate preventive initiatives and funding for relevant development projects. The preventive legitimacy of companies is highly influenced by how well employers apply knowledge to guide practice, and how well employees assess, and experience preventive initiatives as implemented in practice (Van Eerd \& Saunders 2017). To keep preventive legitimacy, companies are challenged to work systematically with scientific knowledge production on what works and under which conditions. In recent years, various research approaches have been applied to pave the way for new preventive insight about workplace interventions and CMD. In this line of work, synthesis research is a central resource (Hannes \& Lockwood 2012). As new research is getting published, synthesis research can help identify systematic flaws between evidence-based knowledge and needs in practice. Here, synthesis research methods are an effective means to keep up with findings that may be relevant to consider and ought to give legitimacy to influence 
company structures and preventive actions. Yet, Nordic research knowledge about work disability management strategies in CMD is still evolving (Andersen 2012; Borg 2010; Vingaard 2015). This study builds on existing synthesis research and looks exclusively at employer strategies to prevent CMD-related work disability. The primary objective is to scope the field of recent research on workplace initiatives and organizational practices to prevent CMD-related work disability, focusing on prevention approaches and interventions strategies applied. Secondary objectives are to discuss CMD-related employer strategies from an integrated prevention approach, which encourage knowledge mobilization and collaborative action with stakeholders outside the workplace.

\section{Methods for achieving review results}

We chose a scoping review method to explore the research field and identify key issues of psychosocial work environment research on workplace initiatives to prevent sickness absence, and work disability management research on organizational policies and practices to promote RTW and outline alternative areas of inquiry. Scoping reviews or scoping studies refer to a process of 'mapping' or 'scoping' the existing literature of a given research area (Arksey \& O’Malley 2005). Scoping reviews are one of several methods used to review primary research (Hannes \& Lockwood 2012), and have been applied widely within work and health research to identify knowledge gaps, summarize findings, and outline new types of research inquiry (Karpur 2014; McDowell \& Fossey 2015; Saunders \& Nedelec 2014). Scoping reviews differ from systematic reviews in that they do not involve a quality assessment of included studies or a checklist for procedures. Scoping reviews are also different from traditional narrative (literature) reviews in that the scoping process requires analytical reinterpretation of the literature (Levac 2010). Arksey and O'Malley (2005) define a scoping review as a process of rapidly mapping the key areas and concepts underpinning a research area to examine the extent, range, and nature of a research activity to identify research gaps and follow-up action. In keeping with Levac and colleagues (2010), scoping reviews are particularly relevant to research areas of emerging evidence, such as workplace initiatives to prevent CMD-related work disability. In this context, a scoping process appears useful because its inductive and inclusive nature allows researchers to incorporate a range of study designs in both published and grey literature, which may support researchers to refine subsequent research inquiries (Levac 2010).

\section{Methodological approach to scoping the field}

Our scoping review methodology builds on a six-step framework for conducting scoping reviews originally developed by Arksey and O'Malley (2005). This study describes the stages of the scoping process with recent enhancement for each step based on the framework by Levac and colleagues (2010):

1. Identifying the research question

2. Identifying relevant studies

3. Selection of studies

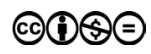


4. Charting and categorizing of data

5. Narrative reporting and summary of results

6. Consultation with practice

\section{Identifying the research question}

The study was commissioned by The Swedish Social Insurance Agency through a developmental initiative between public authorities, scientists, and social partners in working life, focusing on identifying knowledge and development needs related to work and health, sickness absence, and RTW. The research topic for this scoping review stems from a series of dialogue conferences with the stakeholders in this initiative, where the aim was to support employers' strategies for preventing sickness absence, and to promote RTW. We used an iterative process to adapt a set of research specific questions to guide the scoping review process. The following questions have guided the process of scoping the field of recent research on psychosocial work environment and disability management to identify studies on workplace initiatives and practices to prevent CMDrelated work disability:

- What is the extent of knowledge, and key issues in (1) psychosocial work environment research on workplace initiatives to prevent sickness absence due to CMD, and (2) research on organizational practices to promote RTW following CMD?

- What kind of employer opportunities and alternative areas of inquiry emerges from a synthesis that cut across preventive approaches, and reinterprets knowledge from within the two research traditions to inform an integrated CMD prevention approach?

- What types of stakeholders are relevant for bridging preventive initiatives to settings outside the workplace?

\section{Identifying relevant studies}

The following key concepts and terms were used to target the search and select relevant studies for inclusion in the review. Specifically, these concepts include a definition stating what the scoping review means by 'work disability' (Loisel \& Anema 2013), 'psychosocial work environment' (Limborg 2002), 'workplace disability management' (Gensby 2014), 'workplace initiative' (Pomaki 2012), 'sickness absence' (Labriola 2006), and 'return to work' (Krause \& Lund 2004). We decided to do one literature search instead of two separate searches targeting each research discipline, casting a broad net to ensure a comprehensive coverage of the literature in the hopes that we catch a larger percentage of relevant studies. The sources for identifying relevant peer reviewed studies are the electronic databases Medline, EMBASE, PsycInfo, CINAHL, Business Source Premier, Health and Safety Science Abstracts, and Sociological Abstracts. We did consider the Cochrane database as a relevant source of information. We acknowledge that the lack of inclusion of Cochrane database in the literature search could be a potential limitation. However, a recent systematic review conducted by the lead author (Gensby 2012) found no Cochrane reviews published within the CMD group that met the definition 
and eligibility criteria for inclusion in this review. Google Scholar advanced search options was used to search the web to identify unpublished studies. We searched for peer-reviewed papers published in English and/or Scandinavian languages (Danish, Swedish, and Norwegian) between 2000 and 2016. The search concepts and associated key terms are illustrated in a Venn diagram in Appendix 1. Research prior to 2000 was considered informative from a historical perspective but less relevant to inform present preventive initiatives and practices in the workplace. In addition, we used a 'snow balling' approach to scan reference lists of retrieved studies and reviews, which could lead to additional studies, and did a separate search process in three Scandinavianlanguage peer review journals. The targeted Scandinavian-language journals were Journal of Working Life (Tidsskift for Arbejdsliv) (DEN), Journal of Labor Market and Working Life (Tidsskiftet Arbejdsmarknad och Arbetsliv) (SWE), and Spotlight on Working Life (Soekelys paa Arbeidslivet) (NOR). Hand-searching was completed within the Nordic Journal of Working Life Studies. Finally, we contacted a selected group of content experts to suggest relevant studies. The identified articles were combined, using EndNote where titles and abstracts were reviewed to illuminate redundancies.

\section{Selection of studies}

The overall guiding principle for the selection of studies was based on the value of the research findings to map key issues and address knowledge gaps to refine subsequent research inquiries, and not a quality assessment and appraisal checklist for procedure. Using a pre-defined set of inclusion criteria, all studies was independently screened for relevance by at least two review authors based on titles and abstracts. In cases of discrepancy, the authors made contact to discuss issues related to the article until consensus was achieved. If a consensus could not be reached, a third author was consulted. Eligible studies were then read in full text to decide which studies should be included for charting and categorizing of data. The inclusion criteria guiding study eligibility and selection are presented in Appendix 2.

\section{Charting and categorizing data}

The same researchers who worked on the selection of studies (UG, HL, CS, KA) were involved in the data extraction of each study and charting and categorizing of data. In line with Malachowski and Kirsch (2013), we adopted a directed narrative content analysis to capture and compile relevant information and key issues for our data chart into coding categories (Malachowski \& Kirsh 2013). In keeping with Saunders and Nedelec (2014), we used an Excel spreadsheet to chart the general information and terminology used from each article into our coding categories (Saunders \& Nedelec 2014). In total, 10 coding categories emerged in an iterative process, including author name, publishing year, study setting/jurisdictional context, study objective, study design and method, study population, applied prevention approach, type of workplace initiative and organizational practice, intermediate change and/or outcome, and study findings. Since this is a scoping review, no quality assessment was executed of individual studies. Instead, the focus of the data charting process was to map the diversity of study characteristics, 
workplace initiatives, and organizational practices studied to create new insight about the nature of research and provide directions and recommendations for future employer opportunities and studies.

\section{Consultation with practice}

This scoping review was guided by a transdisciplinary (TD) research ideal, which has been promoted over the last decade as a necessary perspective to fully grasp the complexity of CMD-related work disability prevention (Loisel \& Durand 2005). TD may be defined as 'a way to open research beyond any single discipline to seek a more holistic understanding of a problem' (Choi \& Pak 2006). An expectation of TD research in this study is that it contributes to joint problem-solving between researchers from different disciplines to understand preventive approaches and initiatives between the health care provider, the person, the insurance provider, and the employer/workplace (Nowotny 2006). The TD approach adopted for this scoping study included a team of scientists with different disciplinary backgrounds, public-sector decision makers (The Swedish Social Insurance Agency), and businesses (public and private workplace stakeholders). The Triple Helix model (Svensson 2009) was used as a framework to facilitate a joint process to identify relevant research questions and engage stakeholders during the scoping process.

\section{Results of the scoping review process}

\section{Literature searches and selection of relevant studies}

The initial search targeted disability management and psychosocial work environment research in seven electronic databases and identified 1512 potentially relevant records. Our Google scholar search and screening of reference lists resulted in 20 records. Hand searching of the selected nonindexed journals did not yield additional records. After merging databases and removing duplicates, 852 records remained. Following screening of titles and abstracts, 638 records were not peer reviewed research studies, did not focus on sickness absence and/or RTW, and/or did not target CMD-related work disability. In total, 214 studies were included for full text relevance screening. From these, 46 full text articles met our relevance criteria for charting and categorizing of data. Studies excluded from the relevance screening did not focus on workplace initiatives and/or organizational practices to prevent CMD-related work disability in a Scandinavian context. An additional three studies were excluded during data extraction and charting of study data. Finally, a total of 44 studies were included for reporting and data synthesis, including 24 quantitative studies, 15 qualitative studies, and five mixed methods studies. Figure 1 shows the flow chart and steps taken in our search process.

\section{Narrative reporting and interdisciplinary data synthesis}

We present our narrative reporting and data synthesis from scoping the field of workplace initiatives and organizational practices to prevent CMD-related work disability in 
Figure I Flow chart and steps taken in completing our scoping review.

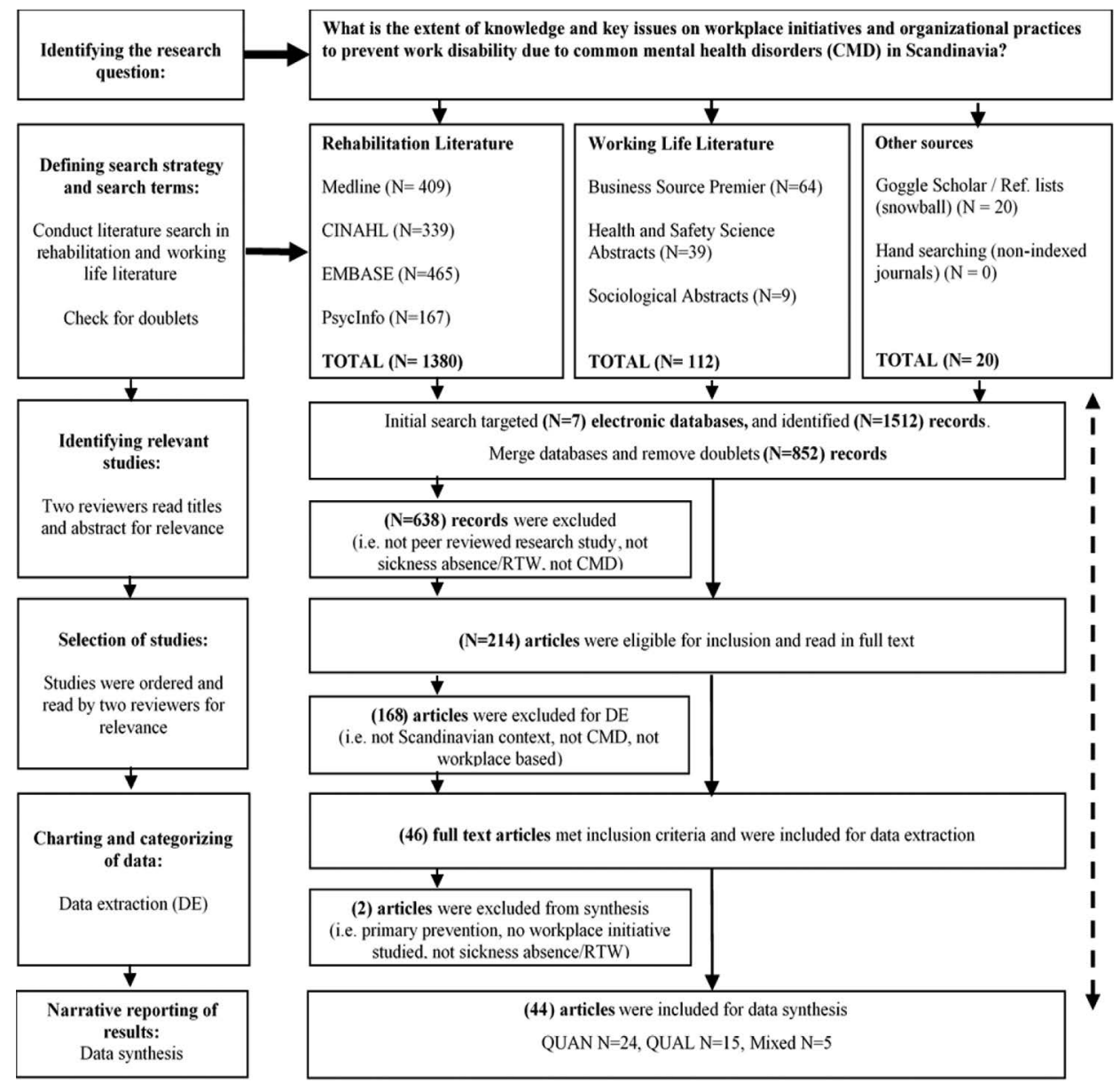

two sections. This first section describes the basic study characteristics and information of the included studies as presented in Table 1 . The second section analyzes the underlying program theory, prevention approach and intervention strategies used, and provide a detailed summary of key issues related to the workplace initiatives and/or organizational practices studied.

\section{Jurisdictional context and setting}

Most of the included studies on workplace initiatives and organizational practices to prevent CMD-related work disability were conducted in Sweden ( $\mathrm{n}=24,54 \%$ ), published between 2003 and 2016. Several authors reported information on the jurisdictional context to understand the role of employers in the Swedish national sickness 
benefit system. A smaller number of studies were conducted in Denmark ( $\mathrm{n}=14,32 \%$ ), published between 2006 and 2016. Many authors described the jurisdictional context to understand the role of employers in the Danish national sickness benefit system. Fewest studies were conducted in Norway ( $\mathrm{n}=6,14 \%$ ), published between 2001 and 2016. Some authors reported information on the jurisdictional context to understand the role of employers in the Norwegian national sickness benefit system.

\section{Study design and methods used}

The typical quantitative data collection methods were survey questionnaires combined with register-based panel data from administrative records or company records. The 24 quantitative studies included the following study designs and methods:

- Five studies used a randomized controlled trial (RCT) design with follow-up periods between three, six, 12 and/or 18 months (Eriksen 2002; Netterstrøm 2013; Peterson 2008a; Reme 2015; Tveito \& Eriksen 2009).

- A variety of quasi-experimental designs were used in 12 studies, including prospective studies evaluating a nonrandomized two-group intervention (Karlson 2010; Martin 2013; Netterstrøm \& Bech 2010; Nielsen 2002). Other studies used a matched-control (Eklund 2013), partial matched control group (Landstad 2001), or matched comparison (Grossi \& Santell 2009; Wästberg 2016; Wåhlin 2012). The quasi-experimental studies included follow-up periods between six months and two years. However, one study used a prospective full-panel cohort, including three cross-sectional samples with a five-year follow up (Borritz 2006).

- The remaining studies adopted case-control designs (Høgelund 2012; Lander 2009), simple before and after design (Anderzen \& Arnetz 2005), prospective cohort designs (Heijbel 2005), or a cross-sectional survey design (Selander 2015).

The typical qualitative data collection methods were semi-structured individual interviews. Some studies use a combination of methods, including individual interviews, focus group interviews, and/or participant observation or participatory workshops. The 15 qualitative studies included the following study designs and methods:

- Most of the studies used a multiple case study design (Braathen 2015; GahnströmStrandqvist 2003; Gunnarson 2014; Holmgren \& Dahlin 2004; Ipsen \& Jensen 2012; Tjulin, 2010, 2011a, 2011b). Two multiple case studies were conducted with matched pairs (Seing, 2015a, 2015b).

- Other studies adopted a narrative case study design (Ståhl 2014), and an action research case study design (Gensby \& Husted 2013).

- Two studies were conducted using ethnographic fieldwork (Larsen 2015; Petersen 2016).

Finally, the five mixed method studies included the following study designs and methods:

- One study was a four-wave questionnaire study with a qualitative case study component (Hjarsbech 2015). 
- Other studies were prospective cohort studies with a qualitative case study component (Andersen \& Westgaard 2013; Nielsen 2012) or a quasi-experimental longitudinal study with added process evaluation (Nielsen 2007).

- One study combined a cross-sectional study with a qualitative multiple case study component (Vinberg \& Landstad 2014).

\section{Setting and participant characteristics}

The included workplace initiatives to prevent CMD-related work disability were defined in relation to different target groups: employers, workers, coworkers, and service providers. Studies focused on various combinations of these groups. The included workplace initiatives were provided in a variety of workplace settings, including the human service sector, the health care sector, the industrial and manufacturing sector, and private consultancies.

The included workplace initiatives and RTW change processes targeted the magnitude of CMD's with work-related stress as the most common mental health problem across the included studies:

- Work-related stress $(\mathrm{n}=19)$,

- Depression ( $\mathrm{n}=13)$,

- Unspecified mental health diagnoses and problems $(\mathrm{n}=12)$,

- Burnout/exhaustion ( $\mathrm{n}=9$ ),

- Anxiety ( $\mathrm{n}=7)$,

- Emotional distress $(\mathrm{n}=3)$.

Some studies did not only exclusively focus on CMD but also included other diagnoses. Further, some studies focused on exploring existing practices, rather than testing interventions.

\section{Outcome measures and change processes studied}

Five groups of outcome measures were identified across the included quantitative studies:

1. The first group of outcomes include individual measures of sickness absence, such as the duration of sickness absence (Andersen \& Westgaard 2013; Anderzen \& Arnetz 2005; Borritz 2006; Eklund 2013; Eriksen 2002; Landstad 2001; Nielsen 2002; Tveito \& Eriksen 2009), number of sick days (Landstad 2001), nature of absence (short or long term) (Landstad 2001), and part-time sick leave (Høgelund 2012).

2. The second group of outcomes include subjective health measures, such as subjective health complaints (Eriksen 2002; Tveito \& Eriksen 2009), self-rated symptom levels and health (Anderzen \& Arnetz 2005; Grossi \& Santell 2009; Netterstrøm 2013; Nielsen 2002; Wästberg 2016), perceived job stress (Eriksen 2002; Nielsen 2002, 2007; Wästberg 2016), personal coping (Tveito \& Eriksen 2009), perceived change in mental health and well-being (Peterson 2008a; Reme 2015), self-assessed 
functioning and work ability (Ahlstrom 2013; Wåhlin 2012), and perceived changes in health-related quality of life (Reme 2015; Wästberg 2016).

3. The third group of outcomes include various $R T W$ measures, such as individual expectations to RTW (Selander 2015), self-efficacy (Wåhlin 2012), initial RTW (Ahlstrom 2013; Karlson 2010; Netterstrøm \& Bech 2010; Selander 2015), rate of RTW (Grossi \& Santell 2009; Netterstrøm 2013), duration until RTW (Høgelund 2012, 2014; Lander 2009; Martin 2013), work participation (Reme 2015), return to pre-illness employer or new employer (Høgelund 2014), and sustained RTW (Karlson 2014).

4. The fourth group of outcomes include various measures related to the work environment, such as perceived worker role (Eklund 2013), workplace characteristics (Borritz 2006), self-assessed work environment (Andersen \& Westgaard 2013; Eklund 2013), perceived quantitative work demands (Peterson 2008a), and/or psychosocial work demands (Tveito \& Eriksen 2009, Vinberg \& Landstad 2014), job satisfaction (Borritz 2006; Nielsen 2002, 2007), perceived change in work conditions (Andersen \& Westgaard 2013; Nielsen 2007; Peterson 2008a; Wästberg 2016), social support (Heijbel 2005), quality of contact (Selander 2015), and type of intervention received (Heijbel 2005).

5. Finally, the fifth group of outcomes include various measures of labour market status, such as employment status (Lander 2009; Martin 2013), employment duration (Høgelund 2014), relapse to absence (Karlson 2014), employee turnover (Borritz 2006), early retirement (Borritz 2006), and costs (Reme 2015).

Four dimensions related to the change processes studied were identified across the included qualitative studies;

1. The first dimension relates to the nature of coordination external to the employer during the RTW process, such as administrative processes and early contact during RTW (Seing 2015b; Tjulin 2011a), management assessment and ability for stakeholder corporation (Gunnarson 2014), and dilemmas of social responsibility in RTW activities (Seing 2015a).

2. The second dimension includes work-related obstacles for RTW, such as environmental and psychosocial barriers for RTW (Holmgren \& Dahlin 2004; Nielsen 2012; Ståhl 2014), work intensification and obstacles at work (Gunnarson 2014; Hjarsbech 2015; Larsen 2015; Seing 2015b).

3. The third dimension encompasses workplace social support, such as the nature of work accommodation and social relations when back at work (Braathen 2015; Larsen 2015; Tjulin 2010), co-worker roles, and capability to support RTW arrangements and initiatives (Petersen, 2016).

4. The fourth dimension includes personal experience of capacity to work, such as experienced change in work ability (Braathen 2015), process of occupational adjustment, and management of recovery at work (Gahnström-Strandqvist 2003; Hjarsbech 2015; Ståhl 2014).

5. Finally, the fifth dimension relates to organizational learning, such as disability management policy development (Gensby \& Husted 2013; Tjulin 2010), capacity building for joint learning and action (Gensby \& Husted 2013; Tjulin 2015), and organizational options and (re)-design factors (Ipsen \& Jensen 2012). 


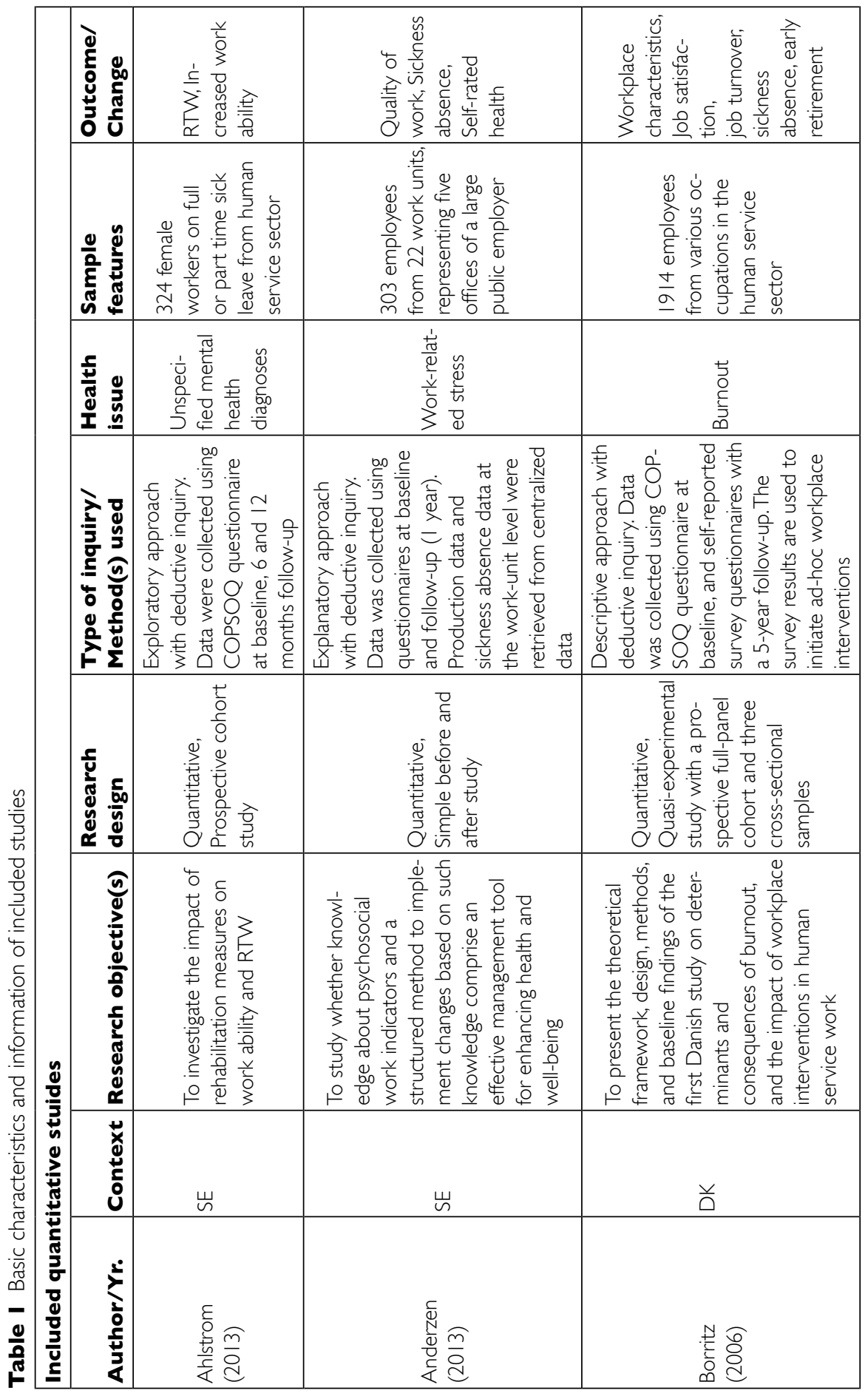




\begin{tabular}{|c|c|c|c|}
\hline 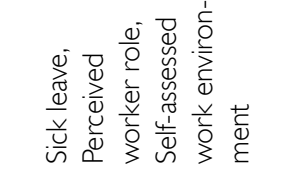 & 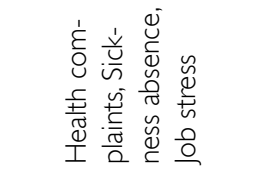 & 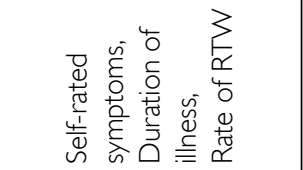 & 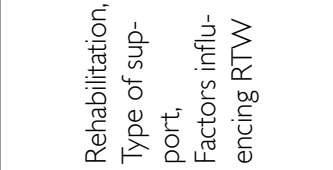 \\
\hline 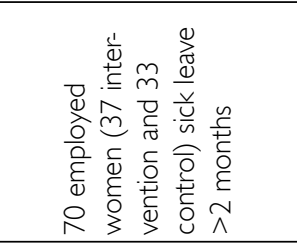 & 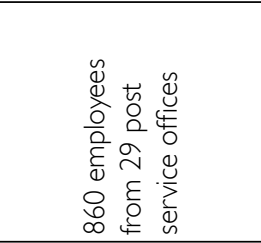 & 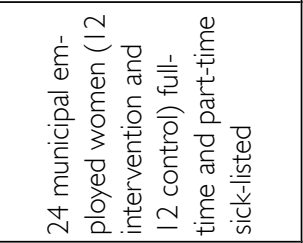 & 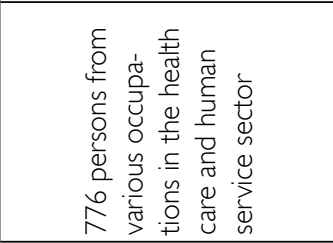 \\
\hline 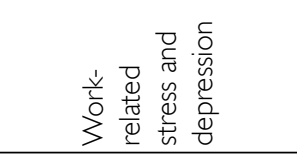 & 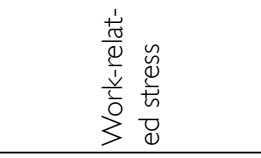 & 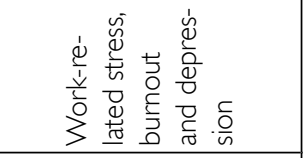 & 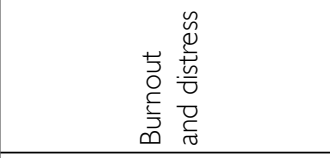 \\
\hline 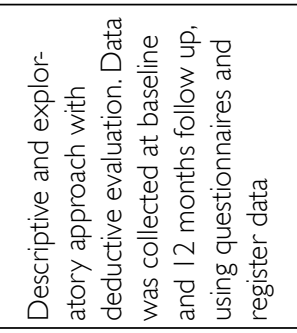 & 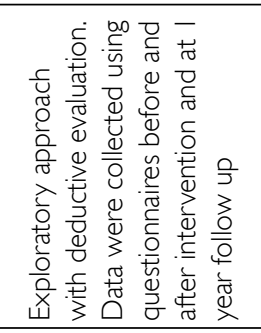 & 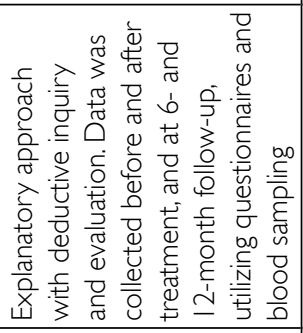 & 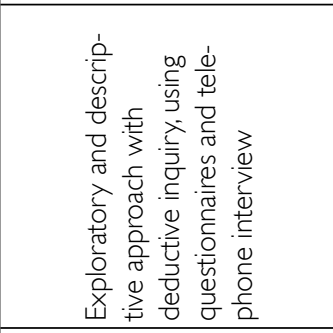 \\
\hline 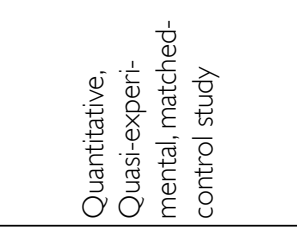 & 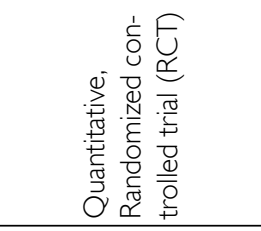 & 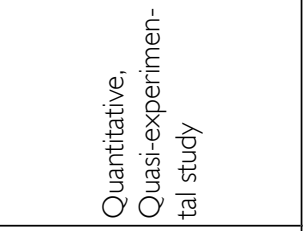 & 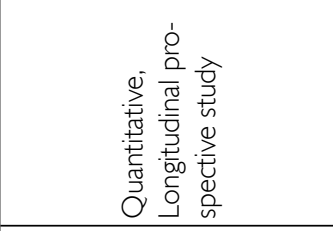 \\
\hline 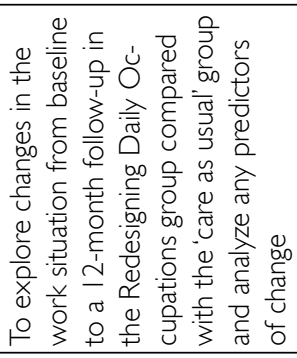 & 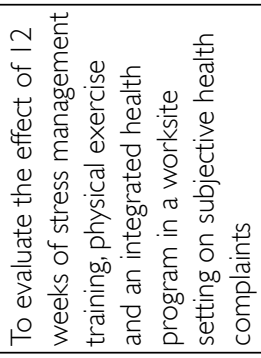 & 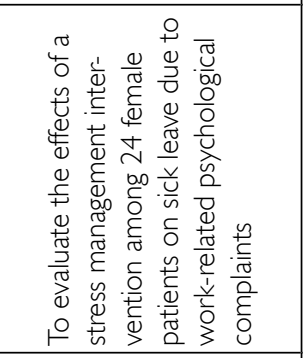 & 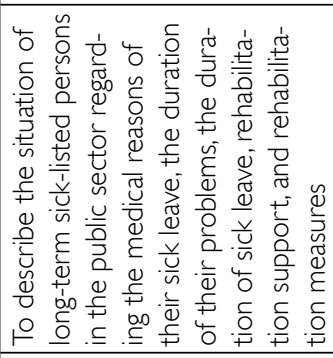 \\
\hline 山 & $\stackrel{\circ}{z}$ & 岃 & 山 \\
\hline 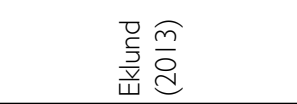 & 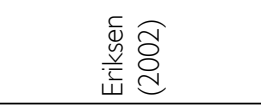 & 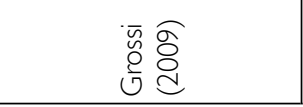 & 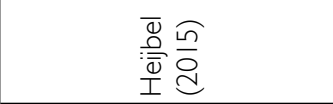 \\
\hline
\end{tabular}




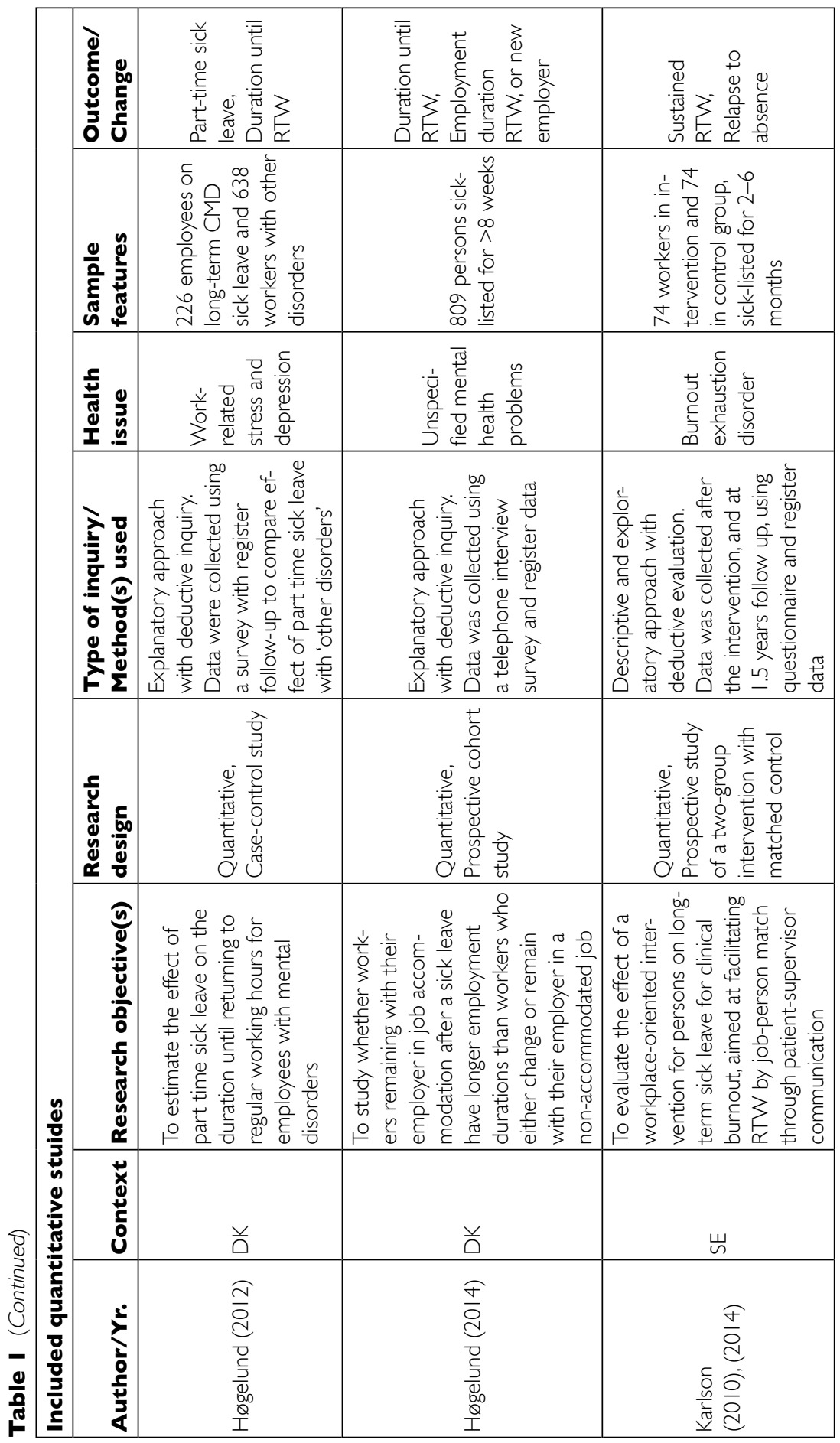




\begin{tabular}{|c|c|c|c|}
\hline 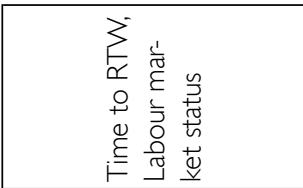 & 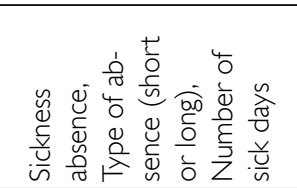 & 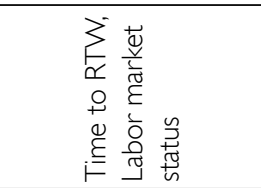 & 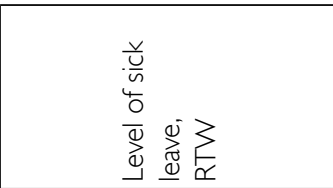 \\
\hline 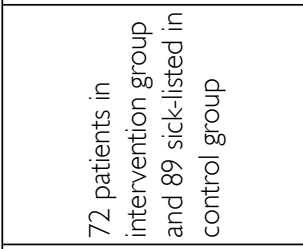 & 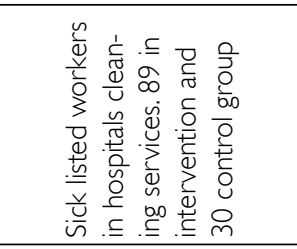 & 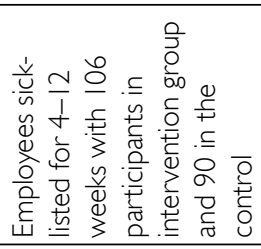 & 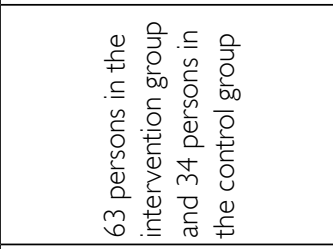 \\
\hline 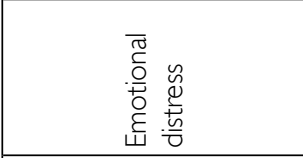 & 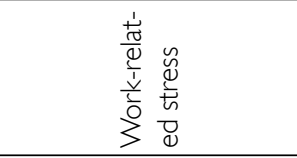 & 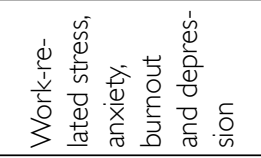 & 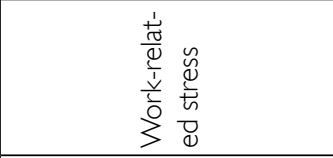 \\
\hline 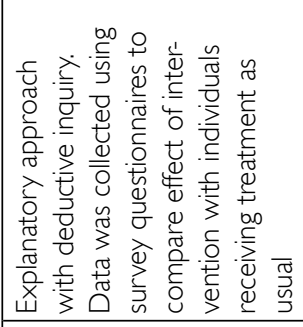 & 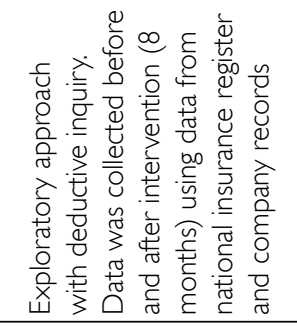 & 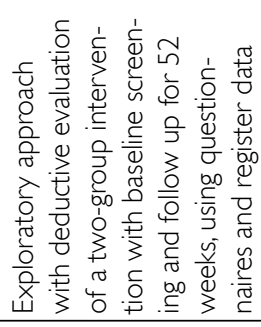 & 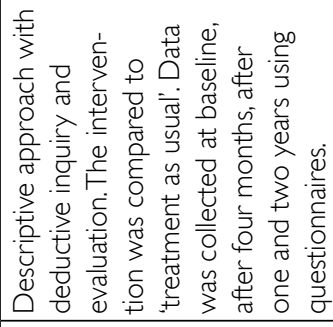 \\
\hline 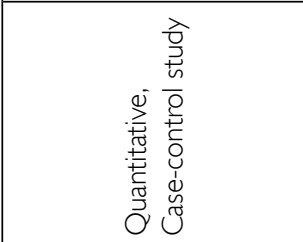 & 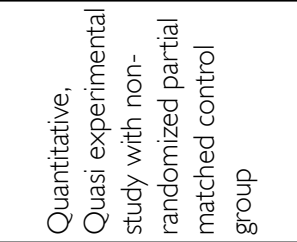 & 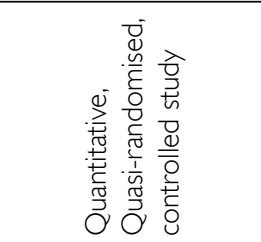 & 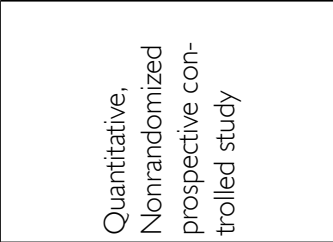 \\
\hline 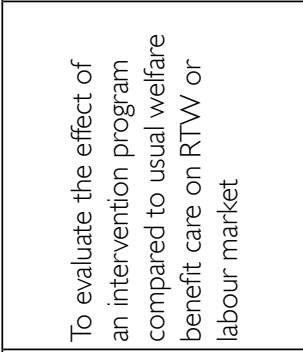 & 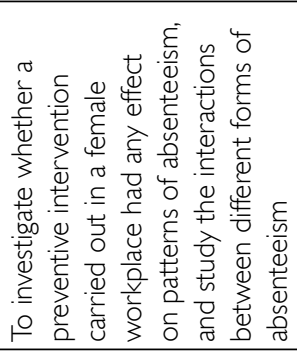 & 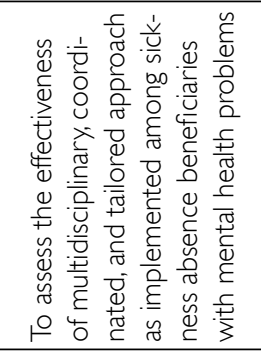 & 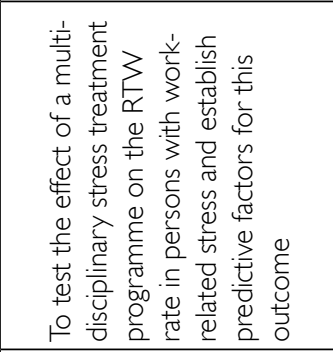 \\
\hline 虫 & $\stackrel{0}{z}$ & 首 & 首 \\
\hline $\begin{array}{l}\text { बे } \\
\text { d } \\
\frac{\bar{d}}{0} \\
\frac{0}{\tilde{c}}\end{array}$ & 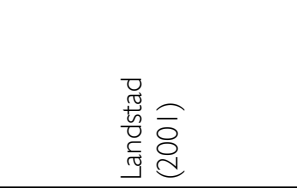 & 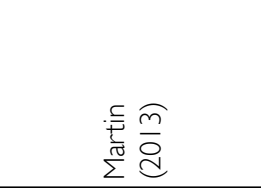 & 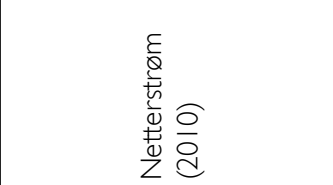 \\
\hline
\end{tabular}




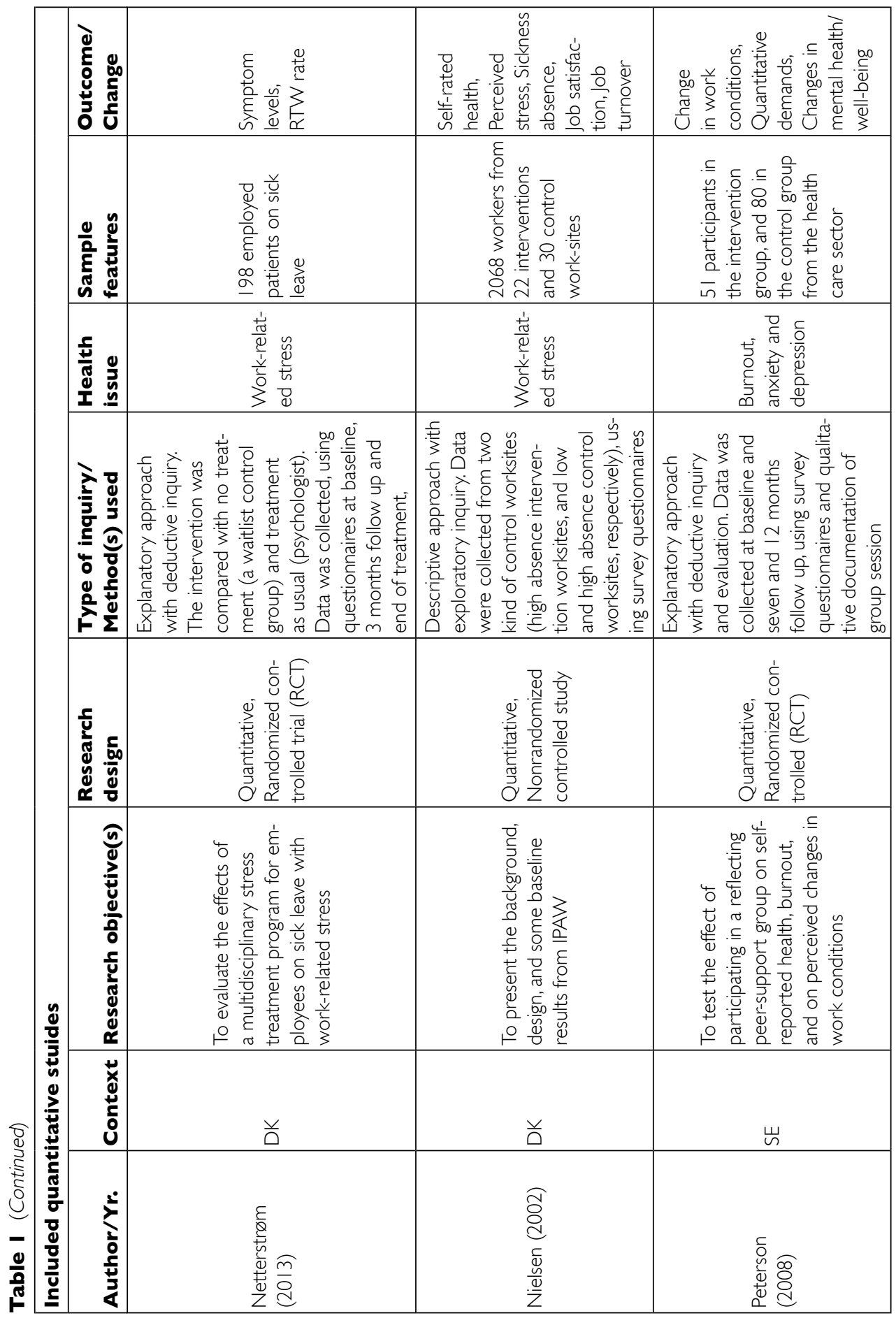




\begin{tabular}{|c|c|c|}
\hline 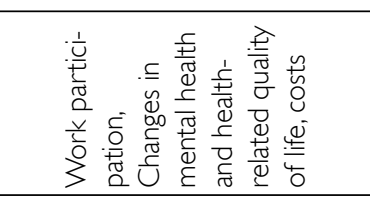 & 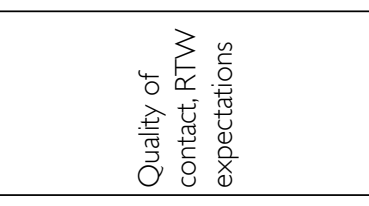 & 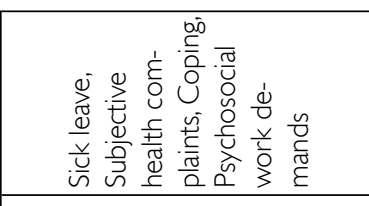 \\
\hline 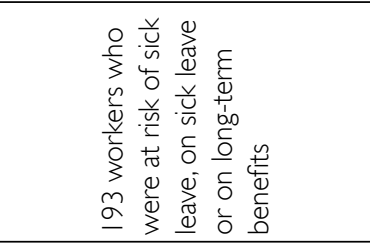 & 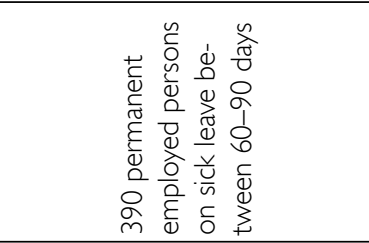 & 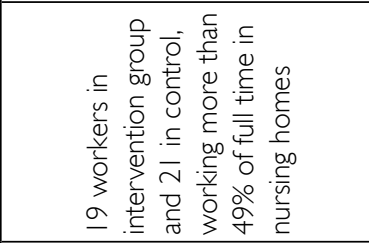 \\
\hline 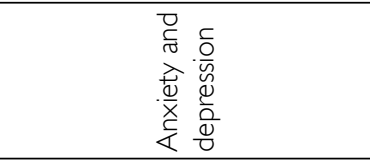 & 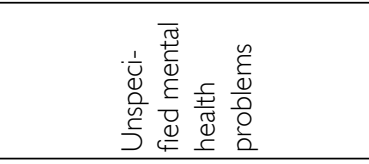 & 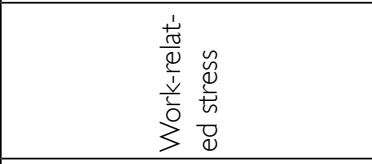 \\
\hline 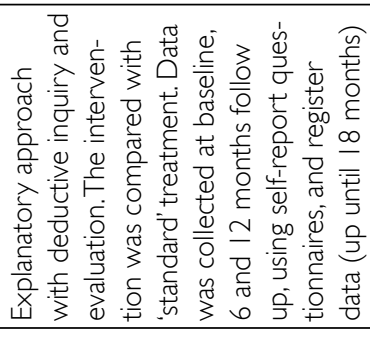 & 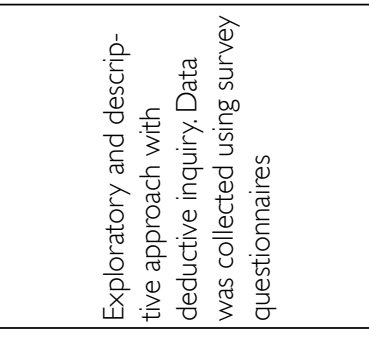 & 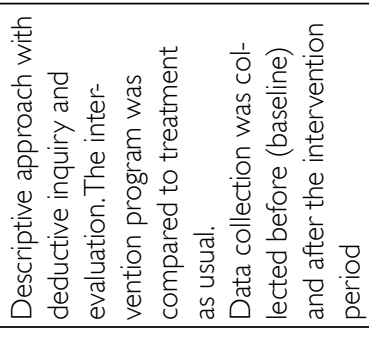 \\
\hline 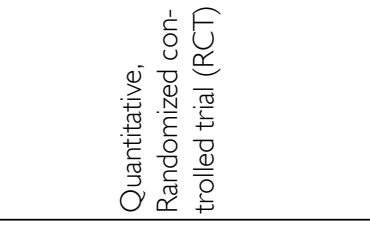 & 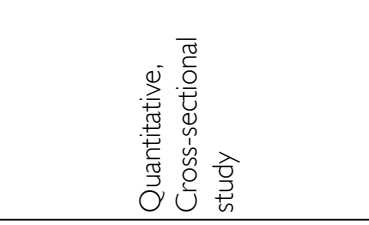 & 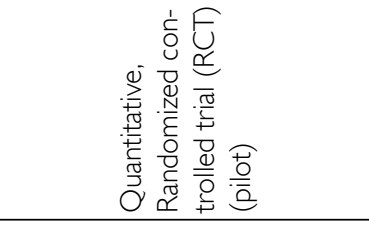 \\
\hline 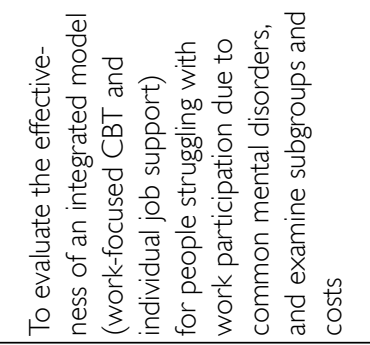 & 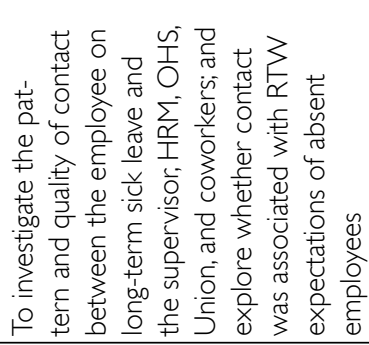 & 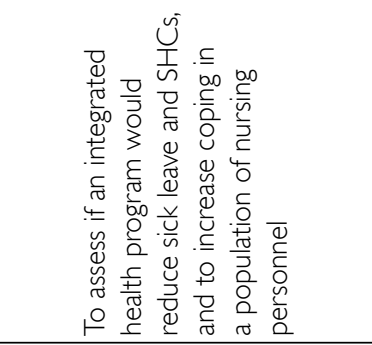 \\
\hline$\stackrel{\circ}{z}$ & 岃 & $\stackrel{\circ}{z}$ \\
\hline 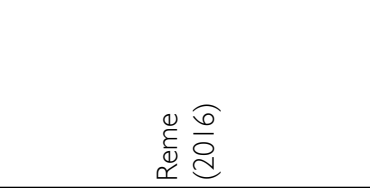 & 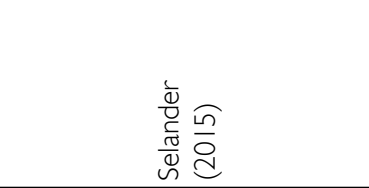 & 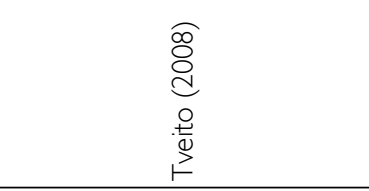 \\
\hline
\end{tabular}




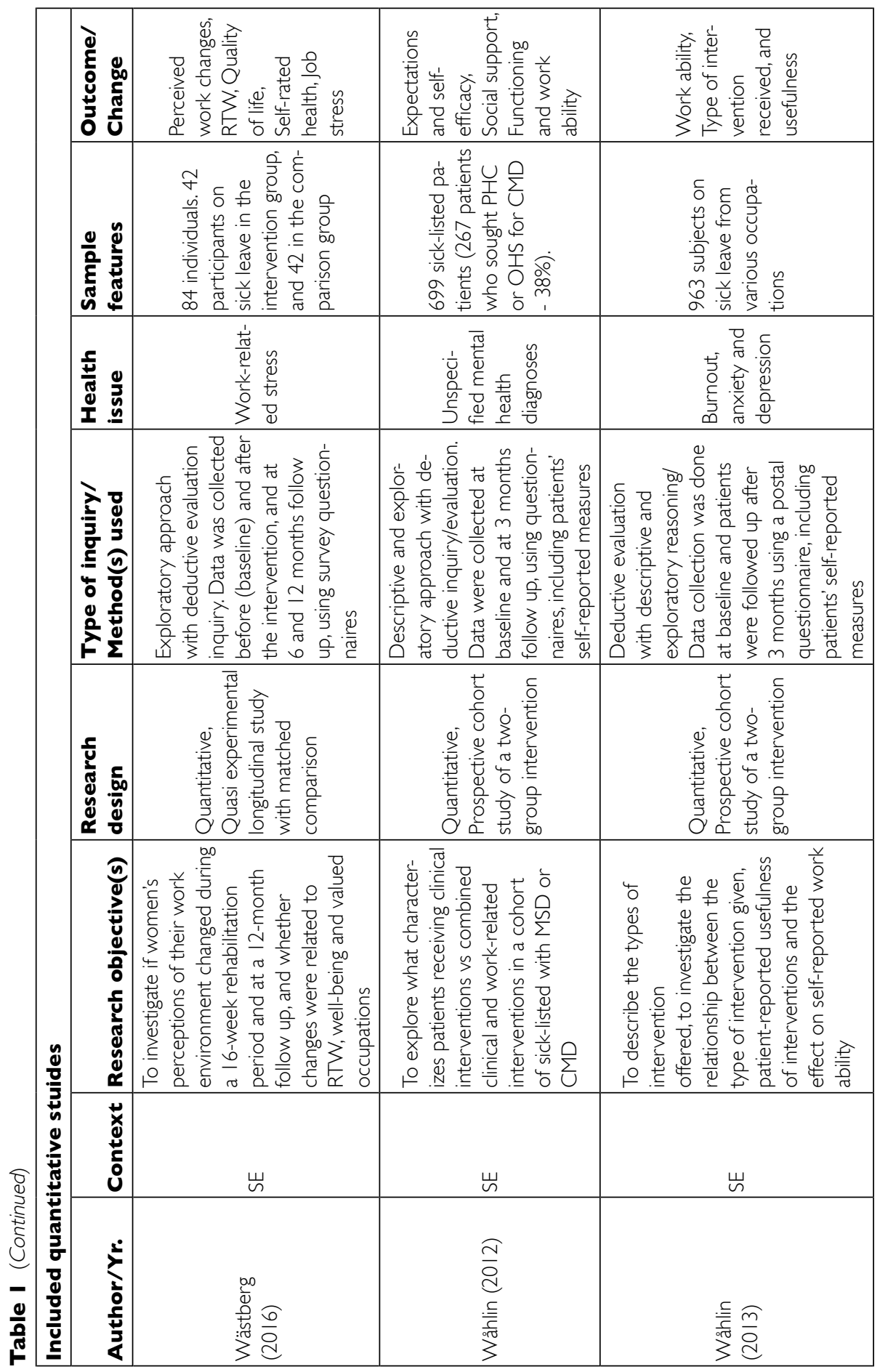




\begin{tabular}{|c|c|c|c|c|c|}
\hline & 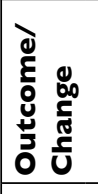 & 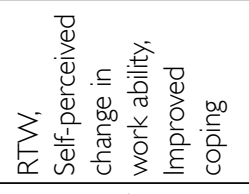 & 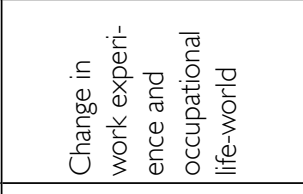 & 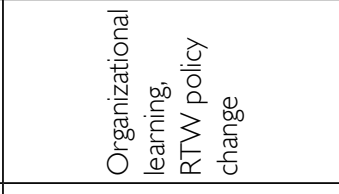 & 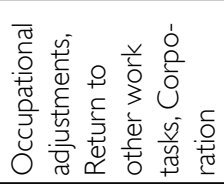 \\
\hline & ๑气 巳 & 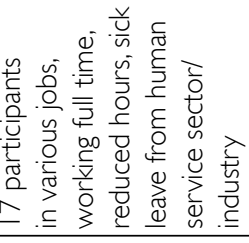 & 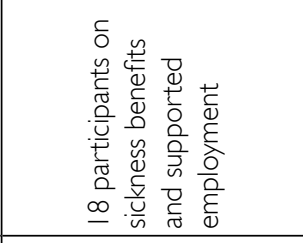 & 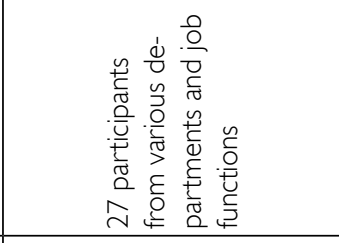 & 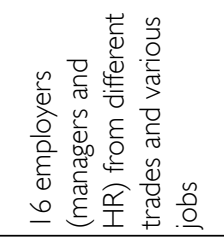 \\
\hline & 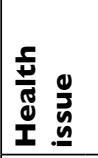 & 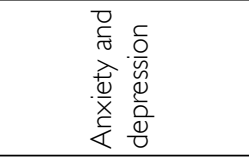 & 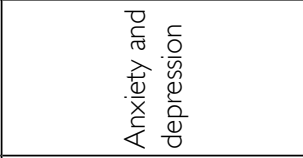 & 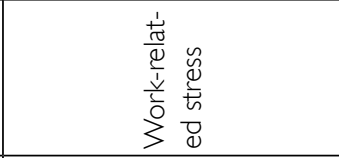 & 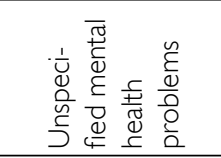 \\
\hline & 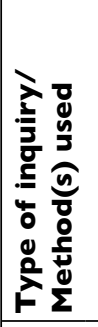 & 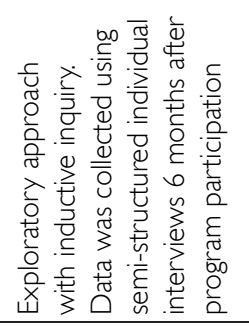 & 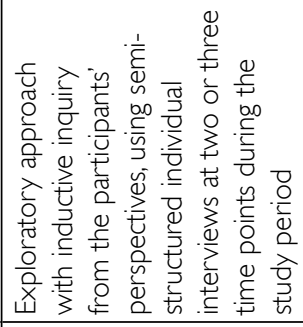 & 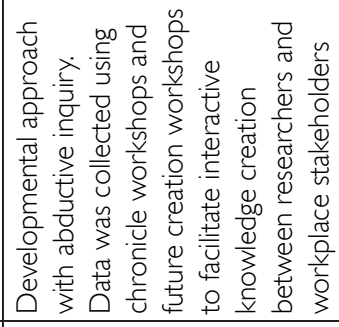 & 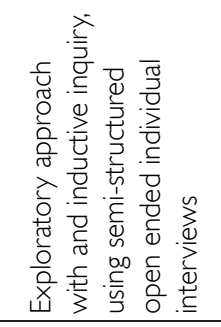 \\
\hline & 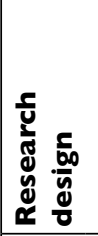 & 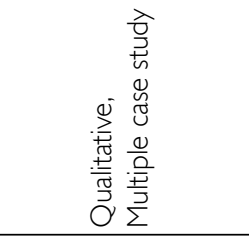 & 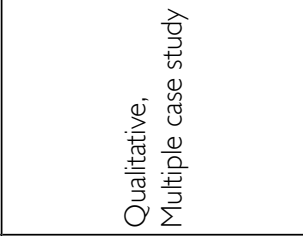 & 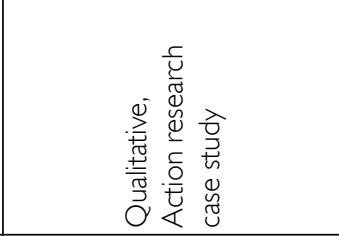 & 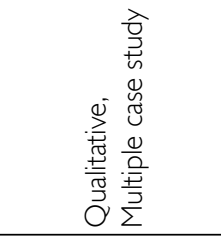 \\
\hline$\stackrel{\boldsymbol{y}}{\underline{\underline{u}}}$ & 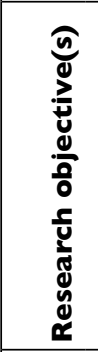 & 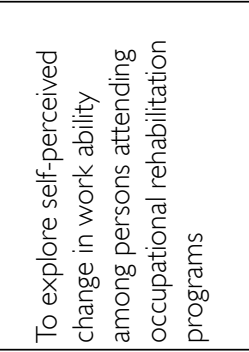 & 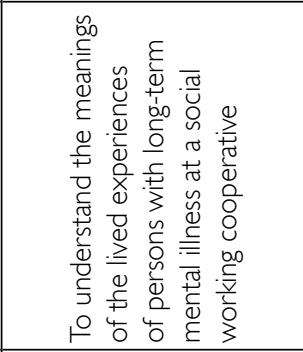 & 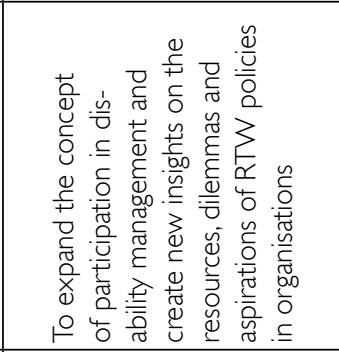 & 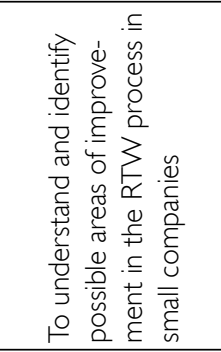 \\
\hline 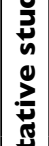 & 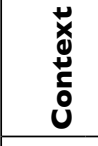 & $\stackrel{\circ}{z}$ & 岕 & 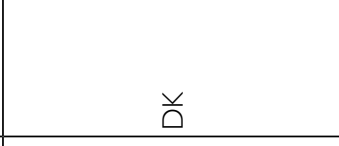 & 岕 \\
\hline 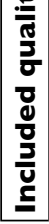 & 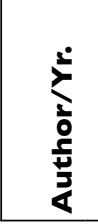 & 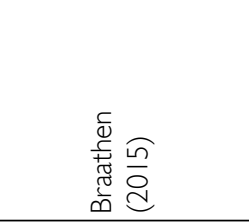 & 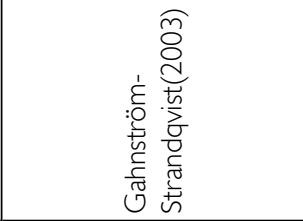 & 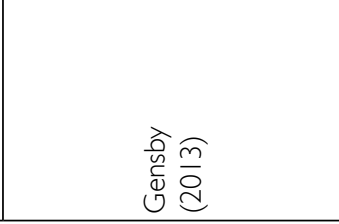 & 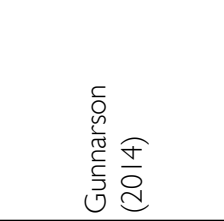 \\
\hline
\end{tabular}




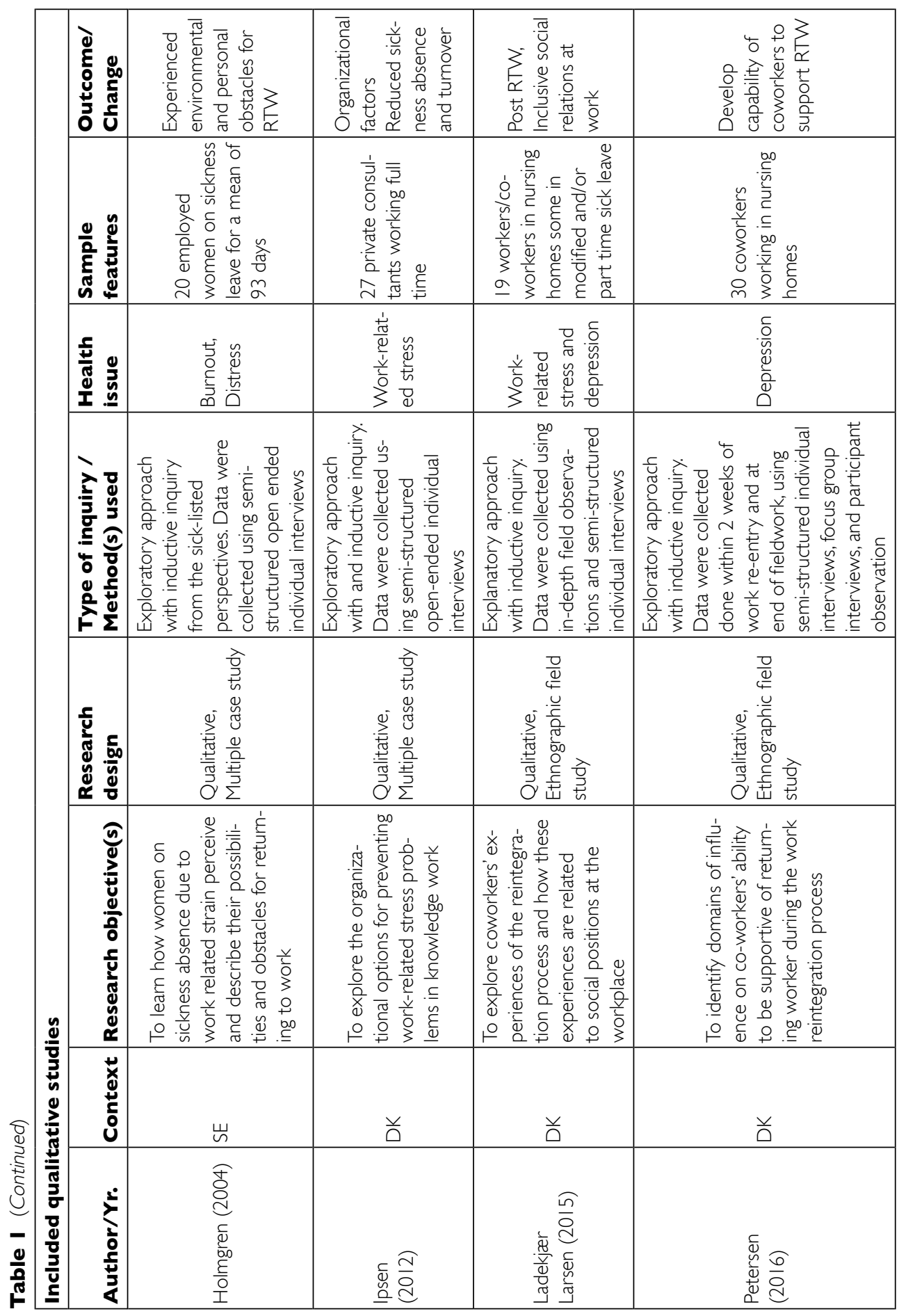




\begin{tabular}{|c|c|c|c|}
\hline 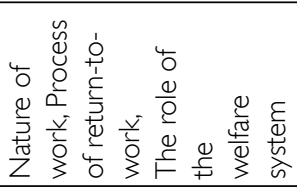 & 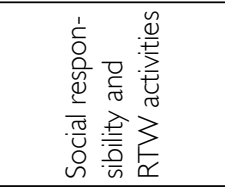 & 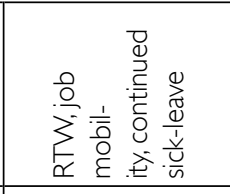 & 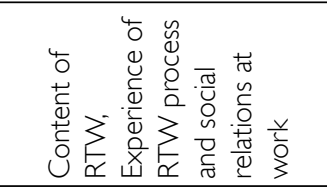 \\
\hline 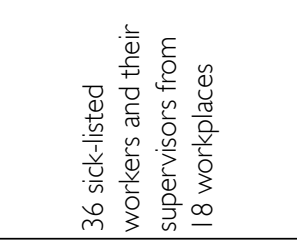 & 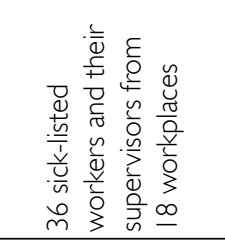 & 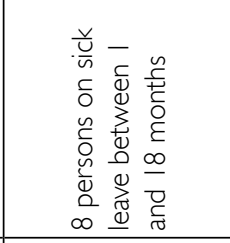 & 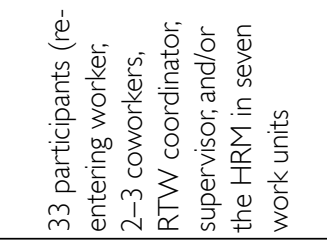 \\
\hline 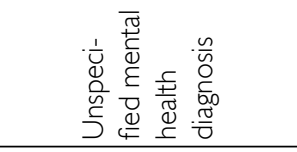 & 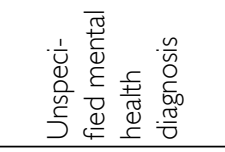 & 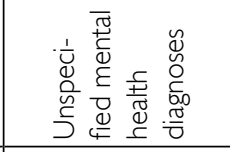 & 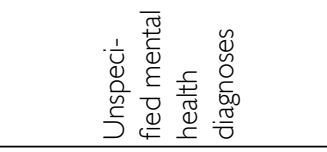 \\
\hline 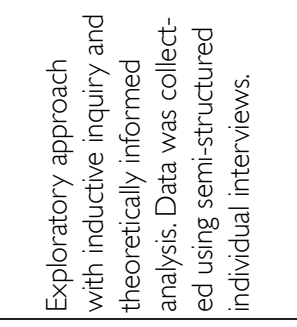 & 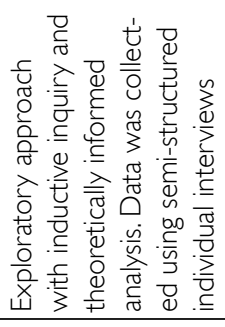 & 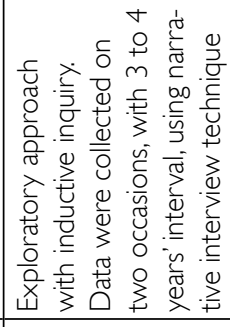 & 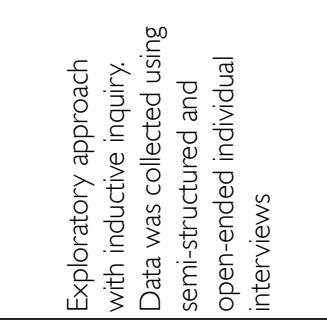 \\
\hline 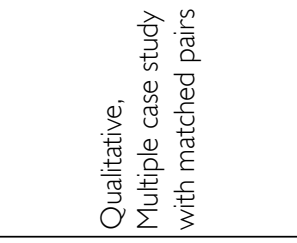 & 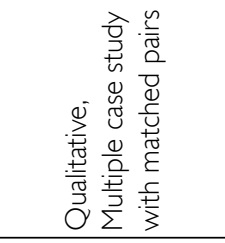 & 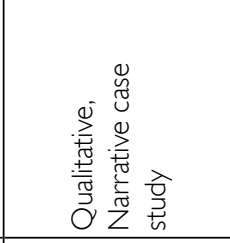 & 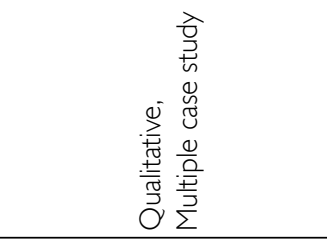 \\
\hline 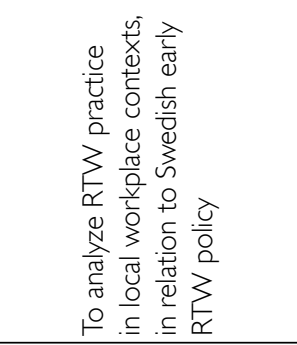 & 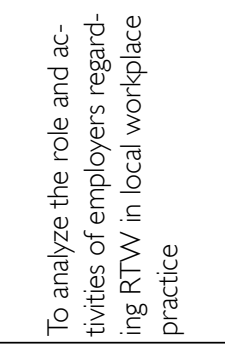 & 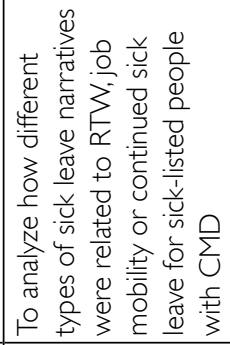 & 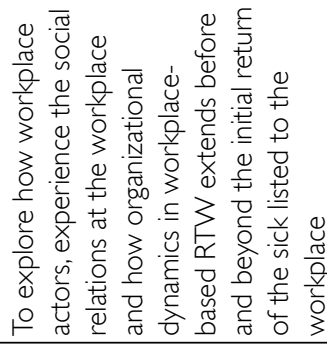 \\
\hline 山 & 山 & 岕 & 岕 \\
\hline 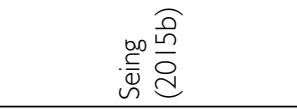 & 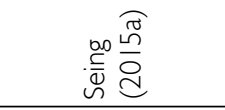 & 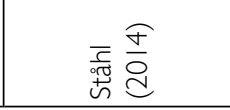 & 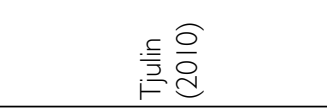 \\
\hline
\end{tabular}




\begin{tabular}{|c|c|c|c|c|}
\hline \multicolumn{2}{|r|}{ 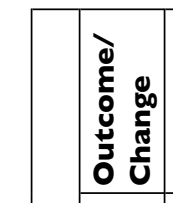 } & 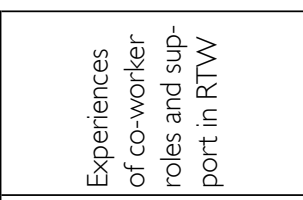 & 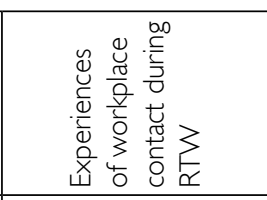 & 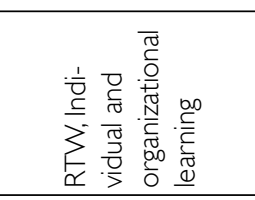 \\
\hline & 을 & 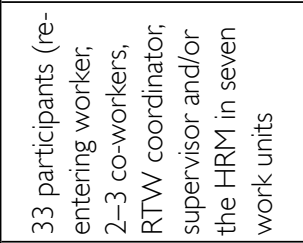 & 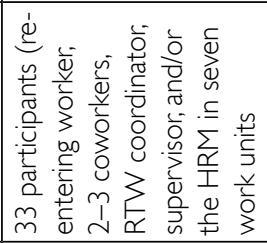 & 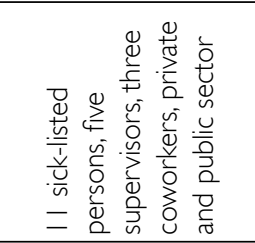 \\
\hline & 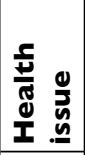 & 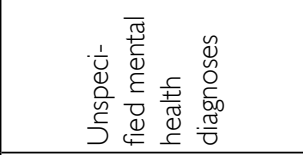 & 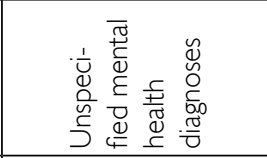 & 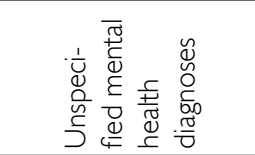 \\
\hline & 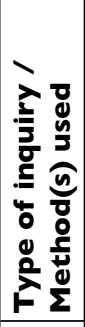 & 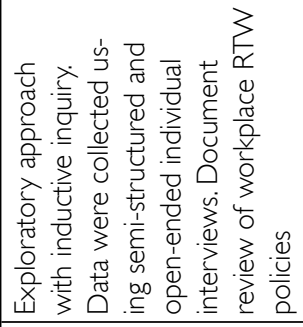 & 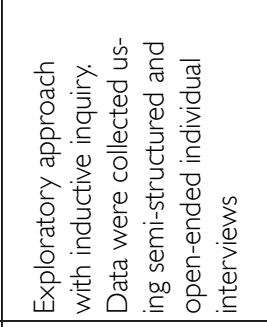 & 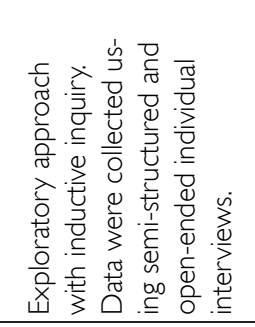 \\
\hline & 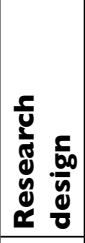 & 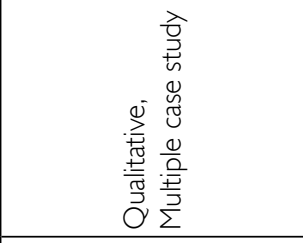 & 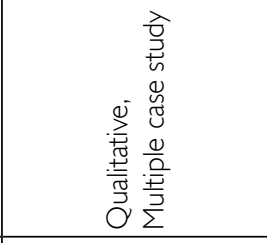 & 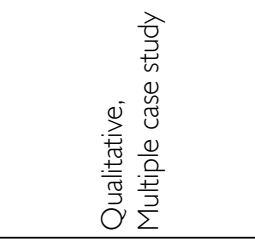 \\
\hline 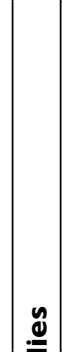 & 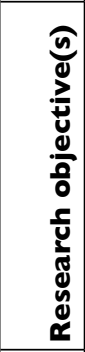 & 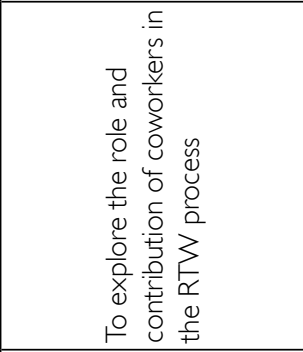 & 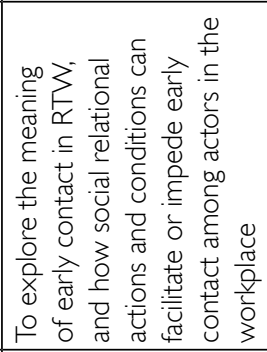 & 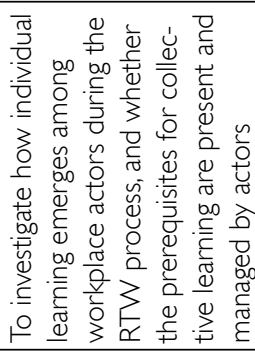 \\
\hline 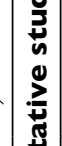 & 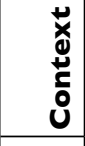 & 虫 & 岕 & 岃 \\
\hline 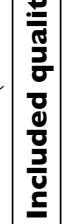 & 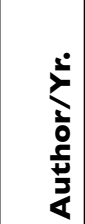 & 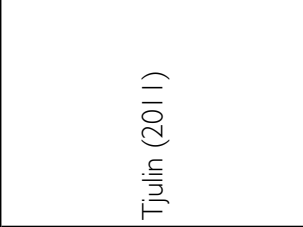 & 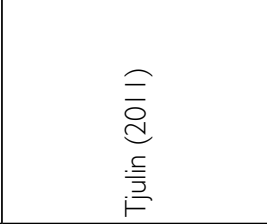 & 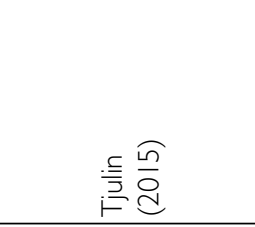 \\
\hline
\end{tabular}




\begin{tabular}{|c|c|c|c|c|}
\hline & 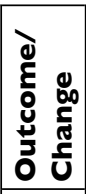 & 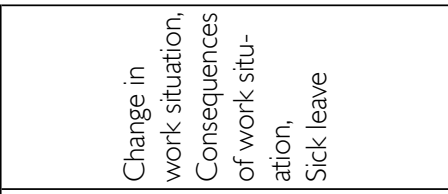 & 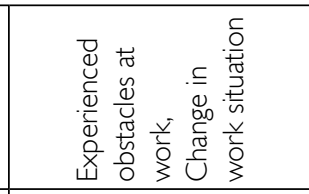 & 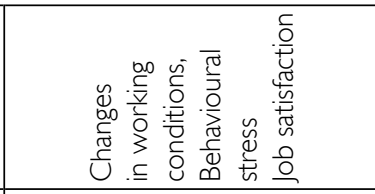 \\
\hline & 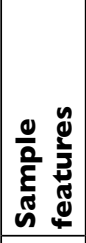 & 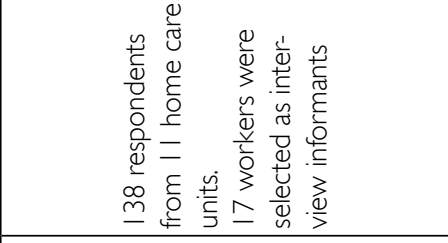 & 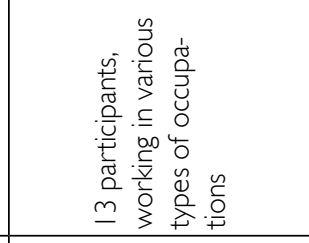 & 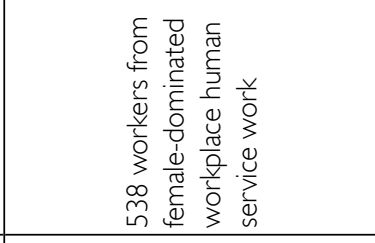 \\
\hline & 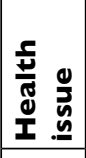 & 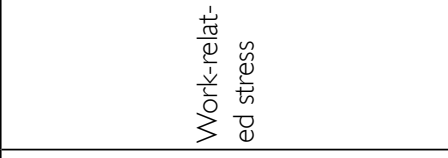 & 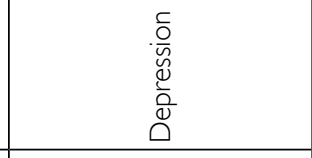 & 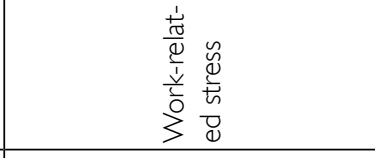 \\
\hline & 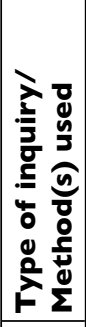 & 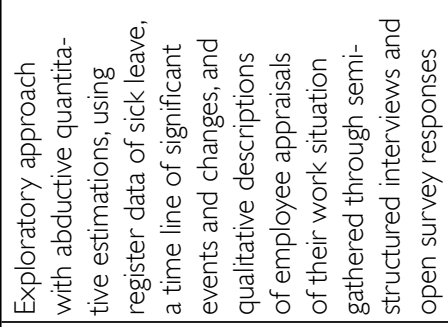 & 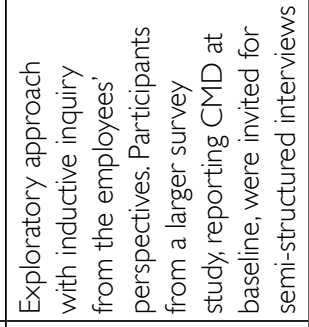 & 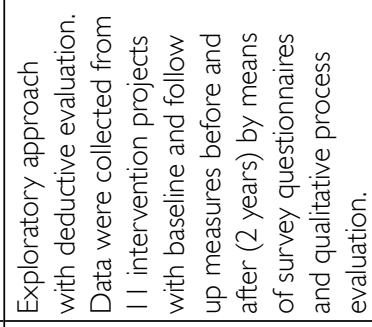 \\
\hline & 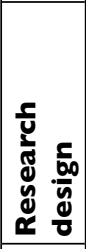 & 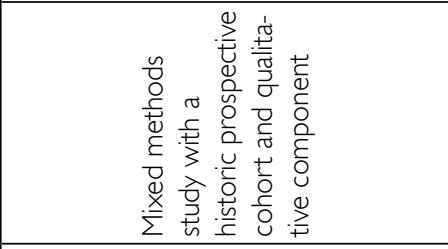 & 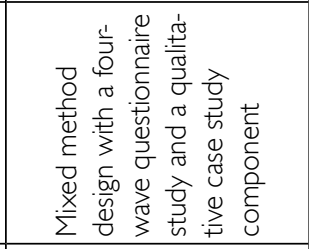 & 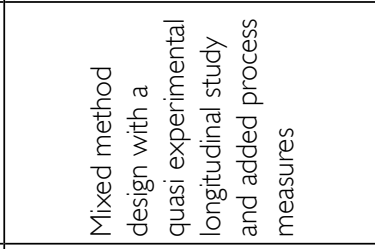 \\
\hline 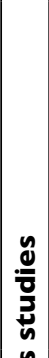 & 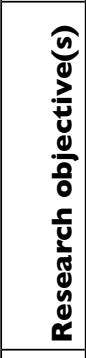 & 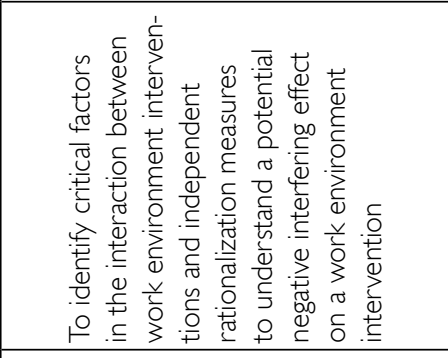 & 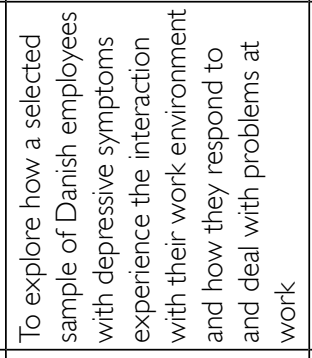 & 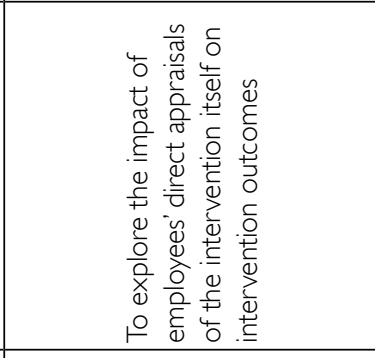 \\
\hline 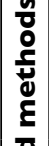 & 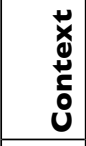 & $\stackrel{\circ}{z}$ & 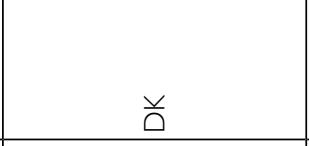 & ט \\
\hline 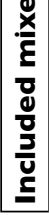 & 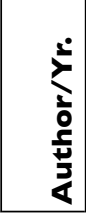 & 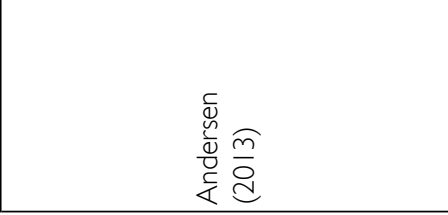 & 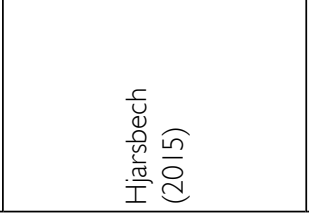 & 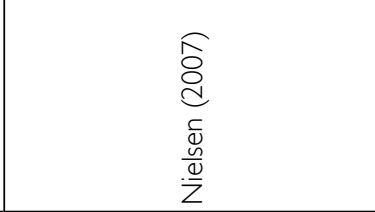 \\
\hline
\end{tabular}




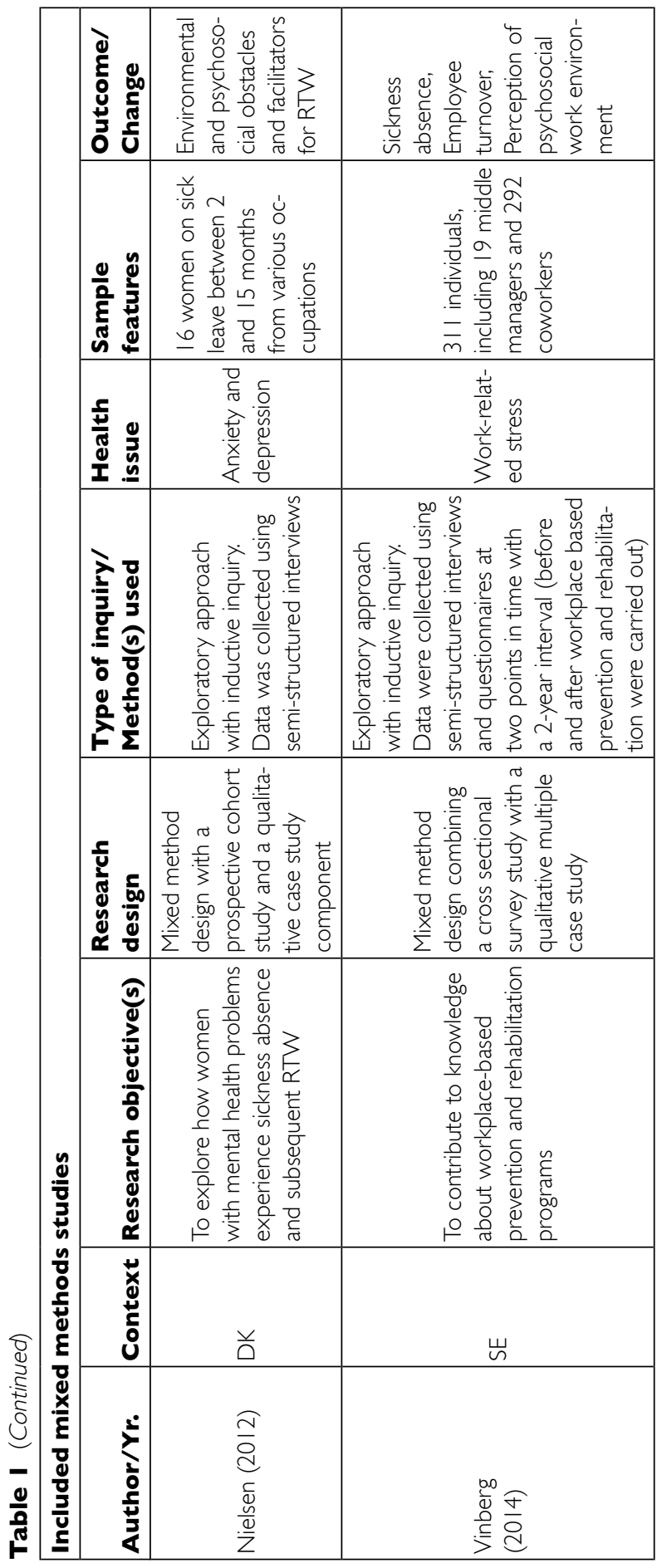

(1) $(9)$ 


\section{Program theories, prevention approach, and intervention strategies}

In this section, we have analyzed the included studies from their own understanding of the prevention approach and the underlying presumptions of how an RTW process can result in successful and sustainable personal and environmental outcomes. This includes an assessment of whether the workplace initiative is considered part of a secondary or tertiary prevention approach, or an integrated approach, and how the aim of prevention is defined. The core of our analysis is the design and execution of the different types of workplace initiatives and the organizational practices that are studied. This includes an overview of the work-related components that constitutes the initiative studied, as well as any key parties and/or stakeholders that have been involved in the execution of the initiative during the RTW process. These questions lead us to look for an overall program theory (Pawson \& Tilley 1997) underlying the descriptions of workplace initiatives/ interventions in the included studies. Based on the included studies (as represented by the mutual research focus on CMD-related work disability), we have uncovered an overall program theory that is the background for almost all the intervention research studies, however specified differently within each study. Some of the qualitative studies do not specify any program theory due to the exploratory nature of the study design. Figure 2 illustrates the overall program theory and the constituent elements identified across the included studies.

Importantly, none of the included studies have presented a comprehensive approach that covers all elements of the program theory; individual studies considered one or more elements in the model. In the following, we will explore the elements of the program theory and give examples of how the elements in the program theory have been applied and prioritized in the included studies.

Figure 2 Program theory for workplace prevention of CMD-related work disability.

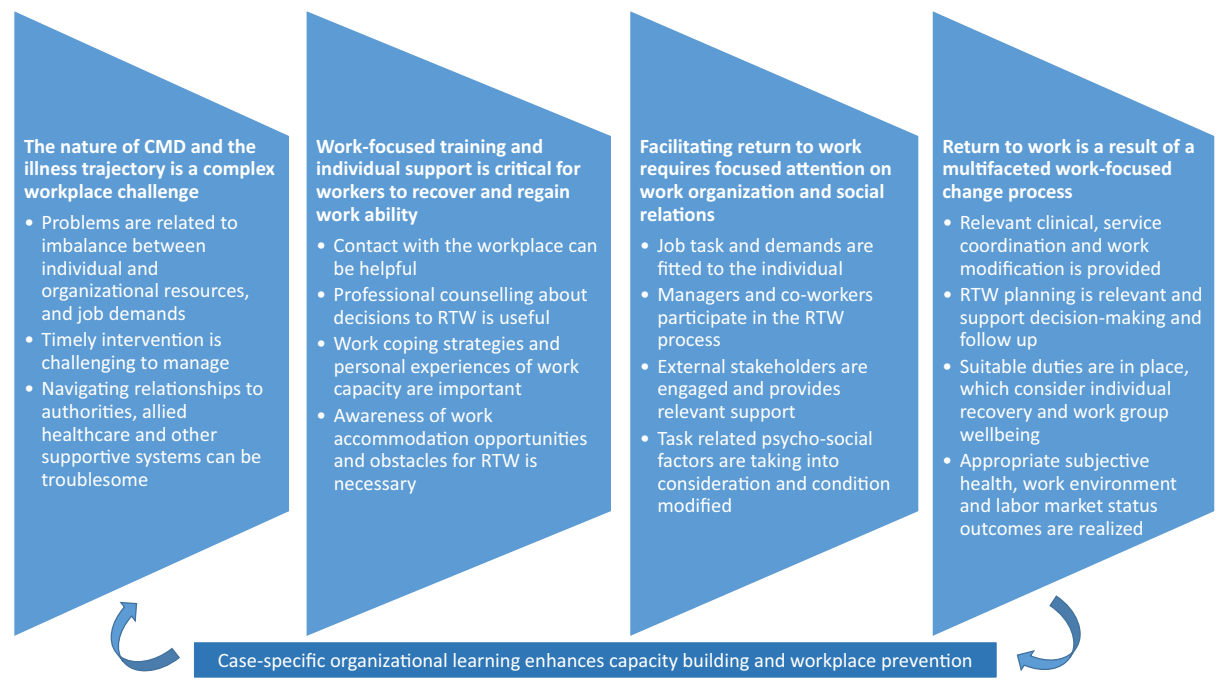




\section{The nature of CMD and illness trajectory is a complex workplace challenge}

The first part of the program theory considers the complex nature of CMD-related sickness absence from the onset of work disability, which can be summarized in the following issues:

- Problems are related to imbalance between individual and organizational resources, and job demands.

- Timely intervention is challenging to manage.

- Navigating relations to authorities and external support can be troublesome.

The scoping of the literature did not yield studies that focus on the relationship between the process before a CMD occur, and the onset of work disability. Included studies seemed to take the prehistory of the CMD as a prerequisite. When prehistory considerations are made, the most common anticipation is that CMD-related work disability stems from an imbalance of individual capacity and resources, and organizational resources and job demands. A substantial epidemiological literature exists on the aspects of the work environment that are risk factors for developing CMD and may predict sickness absence and RTW (Alexanderson 1998; Ekberg 1995; Lund \& Labriola 2009; Vingaard 2015). Understanding these risk factors and the ability to identify and alter them is equally important to consider in workplace prevention programs and rehabilitation (Ekberg 1995).

Many employers consider the nature of CMDs and the illness trajectories a complex managerial challenge where longer periods of time can pass before someone intervenes. This perceived complexity of timely RTW intervention can be conditioned by several issues. One issue is related to the supervisor's behavior toward the sick-listed worker. Here, supervisors can be either too absent or too present, which may result in the worker feeling left alone with the negative experiences of work that led to emotional, cognitive, and somatic symptoms, or in the latter case, that the worker feels controlled and pressured to pursue work resumption (Hjarsbech 2015; Seing 2015a). Another issue concerns the often sensitive information about the worker's health situation, which can make it difficult for supervisors and coworkers to get relevant knowledge to understand work capacity limitations and plan job tasks (Gensby \& Husted 2013; Larsen 2015). Some studies find that timely RTW is a complex measure due to the shifting incentives among workplace actors for initiating interventions and that these incentives often are a mix of obligation and legal responsibility, following the implementation of stricter activation principles in social security insurance (Seing 2015b), social relations in the work group and the supervisor, and/or awareness about individual worker needs (Tjulin 2011a). Here, many employers expect the workers themselves or other parties to be more active in deciding the appropriate time for intervention. Yet again, employers and workers stress the many changes in the work organization as a challenge, since supervisors may be given responsibility for old sick leave cases, in which the worker/health issue in question is not known to the supervisor (Seing 2015b). Importantly, the timeliness of intervention ought to be considered with respect to the recovery of the ill worker, which involves a refocus from negative symptoms to readiness for RTW, while maintaining a positive self-image (Nielsen 2012).

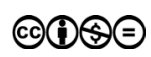


Navigating the relationship to external authorities can be troublesome for employers. Employers explain that they often lack knowledge and are uncertain about their overall role in the RTW process (Gunnarson 2014). Typically, the various stakeholders work alongside each other in subsequent processes, and not as integrated entities with systematic coordination directed toward the employer (Braathen 2015; Martin 2013). This can blur the responsibility for RTW between the various stakeholders and create dilemmas for employers in taking social responsibility for RTW in local workplace practice, which does not adequately prompt employers to engage in RTW (Seing 2015a). Often employers do have knowledge on how to notify and report to the social security agency on the onset of work disability. However, employers propose easier access to facilitate early dialogue around the worker situation and ways to get info about relevant resources and steps in the RTW process. Another critical navigation issue for employers is that the social insurance case manager has knowledge about the workplace and work-related issues that are relevant to consider in the context of RTW. Detailed and better coordinated knowledge that are tailored to the workplace could prompt adequate and timely employer action. Importantly, OHS services could take a more active knowledge broker role in the RTW process, as they are supposed to have the practical workplace insight and understanding of the work context, which can support employers and workers in navigating relations and adequate RTW planning (Gunnarson 2014).

\section{Work-focused training and individual support is critical to recover and regain work ability}

The second part of the program theory represents the workers' situation during the sick leave period, in which the worker considers his/her health situation and recovery, work ability, and possibility to RTW. This part of the program theory comprises studies that focus on various elements related to

- Professional counselling on decisions about RTW is helpful

- Work coping strategies, and experiences of work capacity are important

- Contact with the workplace can be helpful

- Identification of accommodation opportunities and obstacles for RTW are useful

Various professionals can act as helpful experts to support workers with CMD-related work disability about possibilities and decisions to RTW. Particularly, trained psychologists can support the workers' efforts to adopt a problem-solving approach to their problems, provide work-focused cognitive behavioral therapy (CBT), and ensure continuous dialogue with workers' on decisions about RTW and participation in meetings with the workplace (Lander 2009; Reme 2015). In addition to the trained psychologists, social insurance case managers provide advice and support to workers concerning legal matters and the opportunities of various work arrangements (Lander 2009), such as individual placement support (IPS) to facilitate workplace adaptations or identification of appropriate employment (Reme 2015).

Several studies focused on some form of stress management training (Eriksen 2002; Grossi \& Santell 2009; Tveito \& Eriksen 2009; Wästberg 2016). The training 
sessions included different components. In one study, the sessions included therapybased group treatment programs teaching how to identify, understand, and handle psychological and somatic symptoms of stress (Grossi \& Santell 2009). Others included training related to coping with job demands or dealing with the workers own mental health problems, and stress factors or a combination of both (Tveito \& Eriksen 2009). Others focused om awareness training through group-based discussions about everyday work situations and constraints related to perceived psychosocial work demands (Wästberg 2016), or CBT applied in a way that focus on managing CMDs as they relate to work situations (work coping) (Reme 2015). Use of problem-focused strategies was helpful for workers in aiming to redefine and tackle stress-full situations (Eriksen 2002). In some cases, training sessions also involved informal teambuilding and social activities, which should enable coworkers to see each other as a source of social support (Nielsen 2007). Importantly, results show that an integrated model of work-focused CBT and IPS is more effective than usual care in increasing or maintaining work participation for workers' with CMD-related work disability (Reme 2015). Others illustrate how receiving a combined clinical and work-directed RTW intervention resulted in positive outcomes on job satisfaction and well-being (Tveito \& Eriksen 2009), and increased the probability for RTW among patients with CMD, but not among patients with MSD (Wåhlin, 2012, 2013). Furthermore, one study suggests that integrated health programs consisting both physical exercise, information about stress, coping strategies, and practical examination at the worksite have greater effects on the subjective feeling of being in good health than stress management training or physical exercise individually (Eriksen 2002). Although the primary characteristics of stress management interventions were psychoeducative treatment, individual problemsolving methods, and coping strategies in relation to the work situation, interventions varied in their ability to capture the complex ways in which decisions about RTW is influenced by workplace culture or the underlying conditions at work that caused the problem.

Most interventions include some form of contact to the workplace while on sick leave. Contact between the sick-listed and the workplace appears as a well-known recommendation; however, several studies emphasize that the timing and frequency of contact may be less important than the quality (Selander 2015). According to these studies, it is not enough that the contact is established early and frequently. The nature of contact seems equally important to consider, as are the different individual needs for early contact and to be aware of the shifting incentives among workplace actors for making contact (Tjulin 2011a). An additional finding was that supportive organizations such as HRM, OHS, and the unions were considerably less in contact with the worker during the RTW process than supervisors and coworkers (Selander 2015). The studies indicate that unions and OHS services can have a more visible role in the RTW process, and specifically during RTW planning and work practice implementation and change (Gunnarson 2014).

Other interventions include a stronger focus on awareness about obstacles for RTW and the nature of work tasks before sick leave to inform individual RTW planning (Holmgren 2004; Tveito 2009). Typically, dialogue about obstacles for RTW consider possible changes or modifications in the work tasks, or workplace assessment focused on the active input from participants about how to best organize and cope with the work situation (Tveito \& Eriksen 2009). Few studies considered the role of participation a 
mediator for intervention outcomes. One study found that workers appraisal of intervention activities mediated the relationship between participation and reported changes in work conditions, job satisfaction, and behavioural stress. Thus, it seems important to provide timely information and ensure participatory opportunities that leads workers to believe they can influence the content and quality of the RTW activities in a sustainable manner. These results highlight the need to consider participatory and cognitive processes that influence outcomes of RTW interventions (Nielsen 2007).

\section{Facilitating $R T W$ requires focused attention on work modification and social relations}

The third part of the program theory considers the process of facilitating RTW through focused attention on the psychosocial work environment. This part of the program theory illuminates the complex way in which individual capacity to RTW is influenced by the condition of the work environment such as

- Managers and coworkers participate in RTW process

- Work tasks and demands are fitted to the individual

- Task-related psychosocial factors are taken into consideration and condition modified

- External stakeholders provide and coordinate relevant support

The dialogue between supervisors and re-entering workers is thought to be an essential element to sustain RTW and it is therefore suggested that the supervisor could play a more significant role in some of the relatively unseen or grey areas of RTW: the pre-return and the post-return phase at work and role in the workplace prevention (Hjarsbech 2015; Holmgren \& Dahlin 2004; Tjulin 2010). The latter requires that the supervisors manage to be more responsive to employee's experiences of problems and conflicts at work to avoid prolonged sick leave (Hjarsbech 2015). Thus, the relationship with supervisors and the type of social support from supervisors is an important psychosocial work environment issue to consider prospectively (Nielsen 2012). Furthermore, there is a need to involve coworkers in the RTW process and to pay attention to social communication and the negotiation of work roles of the returning worker as well as coworkers (Tjulin 2011b). Hence, acceptance and understanding from coworkers can help social inclusion of re-entering workers in the work group once back at work (Larsen 2015). The work environment and the attitudes toward sick leave constitute an overall frame for how social interaction between coworkers and the returning worker takes place (Larsen 2015; Petersen 2016). Workplace interventions are vulnerable to changes in the work environment, such as negative work exposure from general system rationalization (Anderzen \& Arnetz 2005). The harmful and interruptive effects of, for example, rationalization measures can be prevented by promoting active communication channels among supervisors and workers (Andersen \& Westgaard 2013).

Studies indicate that part-time sick leave by itself is insufficient for promoting the RTW of employees with CMD (Høgelund 2012). Instead, considering alternative placements or work settings can be an important alternative to employment for participants with complex CMD, who do not yet have the capacity to be employed full time in the work community and reflect the need for alternative forms of employment for those who 
wish to return to a job on 'ordinary' conditions. Embedded in this acknowledgment is the understanding that psychosocial rehabilitation can take a very long time to enable persons with complex CMD to develop their personal well-being and individual capacities required to meet new challenges in their everyday working life (Gahnström-Strandqvist 2003). In general, supportive conditions in the psychosocial work environment (such as influence at work, possibilities for development, degree of freedom at work, meaning of work, quality of leadership, social support, sense of community, and work satisfaction) should be integrated in workplace rehabilitation to increase work ability and improve RTW (Ahlstrom 2013). When considering task-related psychosocial factors as part of an RTW intervention employers should aim at some form of condition modification that promote well established psychosocial work environment dimensions such as; high control, high support, high meaning, high predictability, and suitable demands based on a mixture of theory driven and practice-based research between experts' concepts and a participatory input. Facilitating participatory input can be done by using group meetings to address the work tasks and demands in the work group, which has shown to promote individual preparation of RTW with support from the work group. This approach did promote RTW without a change in the workers perceptions of their role in the work group; rather, they renegotiated their view of the work environment to adjust work processes (Eklund 2013).

Different forms of CMD-related work disability ought to be looked at in parallel, with no intervention or external support initiative fit to cover all forms. Here, external service providers are often lacking enough knowledge of the specific background for sickness absence due to work-related CMD (Landstad 2001). Peer support groups using a problem-based approach can be a useful tool to include in service provider programs for alleviating CMD's such as work-related stress and burnout (Peterson 2008b). Consultation between the employer and the worker with joint responsibility for assessing the requirements for rehabilitation, and for initiating measures for an effective rehabilitation plan, addressed to the service provider, seems to promote adequate workplacedirected coordination between those involved in occupational rehabilitation programs (Heijbel 2005). Others have found that an unclear level of employer involvement in a coordinated and tailored occupational rehabilitation program can contribute to a delay in RTW when compared with conventional case management. Thus, understanding the underlying assumptions about how the planned program activities are expected to influence the desired outcomes is useful to assess whether delayed RTW may be a result of either implementation or program failure, or both (Martin 2013). Different data are useful to help strengthen the timeliness of employer involvement during the RTW process. One study combined data of CMD-related work disability and absence duration using models for 'human resource accounting'. By using the model, key sickness absence indicators can be measured on a yearly basis at an individual and aggregated workplace level. In addition, worker input helped inform education and training of line managers during implementation of the model in the workplace (Vinberg \& Landstad 2014).

\section{Return to work is the result of a multifaceted workplace-focused change process}

Overall, it seems that the quality of the psychosocial work environment is closely linked to the possibilities for RTW. Importantly, RTW implies change processes in the work 
organization and social relations, which require attention to individual worker and coworker support needs in all phases of the RTW process. Hence, employer strategies ought to be responsive to changes in the work group or work organization, and that these changes are coordinated in relation to the needs of the re-entering worker and coworkers throughout the RTW process to ensure that the returning worker is not socially excluded as well as ensuring that coworkers are not overburdened in the process. Finally, it seems important to ensure collective space for joint learning and explication of workers' and supervisors' tacit knowledge about obstacles and opportunities at work as means to enhance the dialogue and address the stress management practice (Gensby \& Husted 2013; Tjulin 2015). Often collective and supporting structures that could prevent CMDrelated problems are distant to practical problem-solving, and workers are left with individualized responsibility for own job performance and work situation. A profound development area is to ensure the alignment of a human factor perspective as a central component in the organizational design process in which solutions are managed (Ipsen $\&$ Jensen 2012). Utilizing ongoing case-specific organizational learning seems relevant to enhance RTW capacity building during such development processes (Tjulin 2015).

\section{Discussion and wider implications}

This study represents an attempt to scope the field of Nordic working life research on employer strategies to prevent CMD-related work disability to summarize the existing evidence-base and key research elements in this research area. The results show a wide array of research approaches, considering focus, scope, and methodology. The studies explore practices and test interventions on individual, workplace, and interorganizational levels. However, we found no studies that take a broader perspective that covers all steps in the suggested program theory of the research field (Figure 2). As a result, the studies generally fail to consider several levels of analysis, or to bridge rehabilitating and preventive approaches; the latter is especially obvious, as there is a lack of studies that focuses on causes for sick leave, and how rehabilitative measures can have a preventive effect for other workers.

Based on these findings, we propose developing a multi-stakeholder perspective to encourage knowledge mobilization on how employers can organize rehabilitating and preventive measures, and bridge preventive activities to stakeholders outside the workplace. Given the state of the evidence, employer-oriented approaches to prevent CMDrelated work disability not only seem relevant for improving RTW processes but also to prevent future sick leave. Closer engagement of employers in the development of sustainable preventive activities may also strengthen knowledge exchange between workplaces and welfare services (Ståhl 2010). Employers could be a driver for innovative prevention approaches and processes in relation to professionals in the welfare sector. This scenario would require awareness to employers' decision to insource, cosource, or outsource preventive activities. Among the Nordic countries, responsibility for RTW is often placed outside the workplace, rather than on insourcing or cosourcing systematic work disability management with the workplace as the central arena. The authors argue that this trend has been fuelled by administrative reforms of sickness benefit systems across the Nordic countries, which has not been able to support a shared understanding between welfare professionals and workplace stakeholders about the type of working 
life conditions that are to structure sustainable RTW initiatives at work. In addition, many small businesses do not have established routines or resources to manage intersectorial collaboration (Gunnarson 2014), and do not have a designated RTW coordinator or strong ties with health care providers and/or social insurance providers (Ekberg 2016). Facilitating strong collaborative ties and common understanding around preventive activities is therefore a recurrent challenge for CMD-related work disability prevention. We suggest a renewed focus on how relevant expertise from existing stakeholders involved in occupational health and safety and occupational rehabilitation could be utilized better at an earlier stage.

Figure 3 illustrates the many relevant stakeholders of the occupational health and safety system and the occupational rehabilitation system that may help bridge CMD prevention initiatives to settings outside the workplace. The external occupational health and safety system includes (1) trade safety committee's, (2) occupational health and safety services, (3) the working environment authority, and (4) trade unions and employer associations. The external occupational rehabilitation system refers to four main stakeholders and settings, including (1) the physician and allied health care providers, (2) the social insurance provider, (3) inpatient or outpatient occupational rehabilitation centers, and (4) the occupational medicine clinics. The structure and role of stakeholders may differ across the Nordic countries and system context.

As illustrated in Figure 3, many relevant resources and experiences are not necessarily involved or utilized to help employers develop, adopt, and evaluate workplace initiatives in practice. Here, efforts remain to take into advantage community resources and consider new ways to utilize resources that are already in place among external parties but may not be used or thought of in the context of CMD-related work disability. One promising systematic intervention strategy with the potential to influence successful employer and stakeholder collaboration around CMD-related work disability is

Figure 3 Multistakeholder support model.

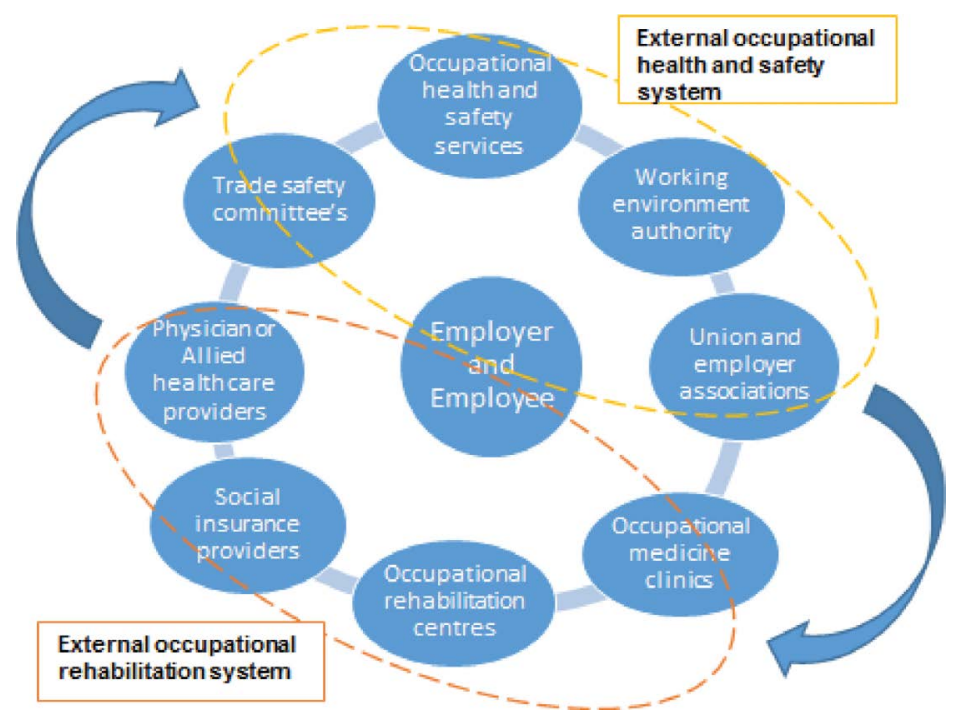


the concept of 'Disability Management' (DM) (Akabas 1992; Habeck 1998; Habeck \& Hunt 1999). DM may be defined as 'a systematic and goal-oriented approach at the workplace which aims to simplify the RTW-process for persons with occupational disabilities through coordinated efforts, taking into account individual needs, workplace conditions and the legal framework' (NIDMAR 2000). ${ }^{3}$ The goal of DM is to create workplace systems of management policies and practices (e.g., WDPM programs) (Gensby 2012), consisting of an integrated set of components promoting safe and timely RTW and sustained job retention within the work environment. A WPDM program therefore relates to conditions of the coordination of work accommodations and practical implementation of RTW activities, and rehabilitative interventions with the agreement of the worker, labor and management, and with the support of occupational health and safety services, allied health care, insurance and service providers, and coworkers.

During the last 20 years, the DM movement has gradually emerged as a way to encourage and support employers to take more control over injury and illness situations and subsequent RTW to promote sustained employment and control unnecessary workers' compensation and work disability cost (Akabas 1992; Habeck 1998). Today, DM is an internationally emerging research area (Geisen \& Harder 2011; Harder 2014). Recently, DM has also manifested itself as a global workplace policy issue, through a 'code of conduct' drafted by the International Labor Organization (ILO), which establishes a set of principles for managing disability in the workplace (ILO 2002). Overall, DM may be understood as an umbrella term, covering a

- Theoretical concept (Geisen \& Harder 2011; Harder 2014)

- Planned intervention/program (Gensby 2012, 2014)

- Counselling practice (Habeck 1994)

- Labour relations strategy (Jodoin \& Harder 2004)

As a theoretical construct, DM remains modestly developed. However, DM may be seen in direct continuation of the work disability prevention paradigm (Loisel \& Anema 2013). Given the multifaceted nature of work disability, planned DM interventions may be delivered by providers, both internal and external to the workplace. This means that interventions may be directed or initiated at the workplace and that the current implementation of these interventions may take place within the workplace-setting or in settings linked to the workplace (Gensby 2014). Employers and decision makers may use the multistakeholder support model to discuss how to create a DM system that balances stakeholder domains against each other to mobilize competencies and collaboration in a way that brings all stakeholders to the table (Hedlund 2007). Such future efforts ought to consider possibilities to unite the different preventive logics and competencies supported by new types of networks and relational coordination to ensure information flow and informed decision making (Gittell 2016). However, this would require interorganizational collaboration to be disciplined and creates new challenging questions about the type of ethical framework guiding the collaboration, the type of economic incentives needed for a fair distribution of economic accountability, and consideration of what parts of a DM system employers could insource, and/or cosource with external parties.

\footnotetext{
${ }^{3}$ https://oshwiki.eu/wiki/Disability_Management
} 
In work disability, innovative thinking and implementation of solutions is highly complicated due to the demands of institutional legal frameworks across health care, social insurance systems, and occupational health and safety. A continuous challenge is therefore to establish collaboration models and partnership initiatives to support employers and public authorities to invest time and resources in the development process. In our view, organizing research and practice partnerships in the context of a Triple Helixmodel (Svensson 2009) could encourage exchange processes and knowledge sharing in which scientists and relevant stakeholders jointly formulate research needs and cocreate analyses and recommendations (Ellström 2008). From such efforts, new opportunities may rise to integrate principles and prevention approaches of occupational rehabilitation, social insurance systems, and the social partners in working life into a coherent DM model that may assist employers in costs control and meaningful service coordination, while protecting individual employability (Harder 2014). Future research also ought to consider how a DM approach aligns with current work environment legislation, and the corporatist approach of the 'Nordic Model', in which collective agreements and industrial relations can guide local policy and practice (Due \& Madsen 2008). The role of collective agreements between labor unions and employers' associations has been studied as a strategy for the prevention of repetitive strain injury (Hasle 2004). However, studies are missing on how to utilize collective bargaining on principles for CMD-related work disability, and ways to use the local labor and management committee as a resource for developing and evaluating workplace initiatives.

\section{Methodological considerations}

The strengths of the study include the interactive research approach. We included various research experts and a diverse group of knowledge users with different backgrounds to give input the research question and discuss findings and implications for future practicebased research, policy and education. Additionally, we conducted a systematic interdisciplinary literature search across two research traditions with the assistance of an experienced research librarian. In practice, empirical evidence about workplace initiatives and organizational practices to prevent CMD-related work disability is rarely synthesized across psychosocial working environment research and DM research traditions to inform new employer preventive opportunities. Adopting a program theory approach in combination with a data-driven synthesis allowed us to carve out the underlying presumptions of how a RTW process can result in successful and sustainable environmental and personal outcomes. This includes an overview of the work-related components that constitute the initiatives as well as which key stakeholders that have been involved in the execution of the initiative, during the RTW process. The limitations of the study include lack of quality assessment of included studies to provide knowledge on what works in practice, which researchers and practitioners ought to consider when judging the program theory. Another issue is the decision to focus on the Scandinavian countries (Sweden, Norway, and Denmark). We are aware that there is a rationale for including all Nordic countries, since all Nordic countries have welfare states based on similar principles and share many elements in their approaches to promote mental health and RTW at the workplace. Thus, including other Nordic countries, such as Finland, could have added additional information to the study. Another potential limitation is the inclusion of studies with participants 
with other diagnoses than CMD, which we considered relevant if they included participants with CMD and met our other selection criteria. This could potentially have obscured the results specific to the CMD group. In addition, some studies did not study specific initiative but were considered relevant since they provided valuable descriptions and exploratory insight to actual employer strategies toward CMD-related work disability prevention and/or RTW processes. Despite having a comprehensive approach to the literature, we cannot be sure that we captured all relevant studies within the scope of the review, and it is possible that the search strategy and selected databases may have impacted the search results. Lastly, we could have used more resources on reaching out to peers and searching the grey literature for relevant peer review studies.

\section{Ethical considerations}

A scoping review is secondary research and therefore exempt from requiring ethical approval. As a review author, you become responsible for providing a 'state of the art' representation of existing work. All steps taken, and criteria used to assess this work, therefore ought to be dealt with in an explicit and clear manner to ensure that data extraction and study details are reported carefully, and that neither data nor essential meaning are lost in the analysis. In addition, synthesis researchers find themselves 'spokesmen' for the research area they review and synthesize. The review team have strived to properly interpret, and acknowledge and cite fellow colleagues who conducted the original work (Petticrew \& Roberts 2006). The review team are aware that commitment is required to publish synthesis results beyond traditional scientific journals to inform the broader research community and ensure proper knowledge transfer and exchange activities.

\section{Conclusion}

Scoping the field of research on workplace initiatives to prevent CMD-related work disability, we identified several key issues that cut across disciplinary preventive thinking. The included studies take up various elements of the program theory and present many valuable enhancements of our knowledge of the RTW process. However, even if some studies conclude that a comprehensive approach, including assessment of the causes to sick leave, individual support, relevant changes in job task, and relevant involvement of managers, coworkers, and external stakeholders are important to develop a strategy to prevent CMD, no studies adopting this type of comprehensive focus were found. There seems to be a lack of studies that include the process leading to sick leave in the study of RTW, which could help broaden the possibilities of early prevention. Focus on how the individual can be empowered to regain health and work ability are documented, as are interventions on workplace level. However, there are limited efforts on how to combine various workplace interventions, and their evaluation. There seems to be an evolving knowledge base on what aspects of the psychosocial work environment employers need to consider in the RTW process to probably accommodate workers with work disability. However, limited knowledge exists on how to implement a preventive approach that consider aspects of the psychosocial work environment in RTW problem-solving among stakeholders outside the workplace. Several different internal and external actors 
and bodies play important roles in the RTW process, but studies that can include the relations and possible coordination and communication between such actors will without doubt enhance our understanding and our ability to propose further development of RTW strategies and practices. Importantly, there seems to be a huge potential in designing orchestrated interventions to consider the timing and content of other intervention programs outside the workplace. Prospectively, decision-makers and researchers could refine research questions to enhance employer strategies and knowledge sharing from a multi-stakeholder support approach that

- Emphasize the important role that the psychosocial work environment seems to play for RTW, particularly among workers with CMD compared with musculoskeletal disorders.

- Use the potential of including stress-prevention in RTW programs such as training and organizational development that are based on an understanding of the relation between the sources of work-related problems and the organizational design of the workplace.

- Consider the wider organizational and managerial elements of a workplace-based RTW approach, such as improving collective social support and the organizational capacity to manage accommodation at work.

- Consult how national RTW programs support the development of organizationallevel interventions at work, including adjustment in work organization, technical improvements, and role clarity among workplace parties.

- Consider ways to promote a supportive relationship between the re-entering worker and the supervisor before RTW planning to facilitate meaningful communication between supervisors and the sick-listed worker during the RTW process.

- Consider how sick-listed workers' readiness for RTW is related to potential problems in the work environment, and the way supervisors respond to making adequate adaptations, both for the individual and for the work group to prevent future work disability.

- Target process challenges related to modification of work hours or work tasks according to individual work capacity, which is a change process that influences the whole organization, including coworkers' everyday working life.

- Consider how collaboration with external authorities and providers cuts across the various phases of the RTW process and what type of knowledge is needed to provide needed resources to achieve desired RTW outcomes.

- Emphasize the value of context tailored interventions focusing on a combination of improving individual capacity and changes in the work conditions based on information of the actual challenges in the work environment.

\section{Acknowledgment and disclosures}

The authors report no conflicts of interests. This work has received financial support from the Swedish Social Insurance Agency. The authors would like to thank the project advisory board, led by Stefan Mörk, for many good discussions in conducting this study. Thanks to Laura Avlund Guldager for assisting in data management and synthesis. 


\section{References}

Ahlstrom, L., Hagberg, M., Dellve, L. (2013). Workplace rehabilitation and supportive conditions at work: a prospective study, Journal of Occupational Rehabilitation 23: 248-260. doi: https://doi.org/10.1007/s10926-012-9391-z.

Akabas, S. H., Gates, L. B., Galvin, D. E. (1992). Disability Management: A Complete System to Reduce Costs, Increase Productivity, Meet Employee Needs, and Ensure Legal Compliance, New York, American Management Association.

Alexanderson, K. (1998). Sickness absence: a review of performed studies with focused on levels of exposures and theories utilized, Scandinavian Journal of Public Health 26: 241-249.

Andersen, G. R. \& Westgaard, R. H. (2013). Understanding significant processes during work environment interventions to alleviate time pressure and associated sick leave of home care workers - a case study, BMC Health Services Research 13: 477-489. doi: https://doi. org/10.1186/1472-6963-13-477.

Andersen, M. F., Nielsen, K. M., Brinkmann, S. (2012). Meta-synthesis of qualitative research on return to work among employees with common mental disorders, Scandinavian Journal of Work, Environment \& Health 38: 93-104. doi: https://doi.org/10.5271/ sjweh.3257.

Anderzen, I. \& Arnetz, B. B. (2005). The impact of a prospective survey-based workplace intervention program on employee health, biologic stress markers, and organizational productivity, Journal of Occupational \& Environmental Medicine 47: 671-682.

Andreassen, T. \& Spjelkavik, O. (2013). Working towards employers: Integrating inclusive working life and labour market policies. [Arbeidet mot arbeidsgiverne: Integrering av inkluderingspolitikken og arbeidsmarkedspolitikken?] Oslo, Gyldendal.

Anema, J. R., Schellart, A. J. M., Cassidy, J. D, Loisel, P., Veerman, T. J, Van der beek, A. J. (2009). Can cross country differences in return to work after chronic occupational back pain be explained? An exploratory analysis on disability policies in a six country cohort study, Journal of Occupational Rehabilitation 19: 419-426. doi: https://doi.org/10.1007/ s10926-009-9202-3.

Arksey, H. \& O'Malley, L. (2005). Scoping studies: towards a methodological framework, International Journal of Social Research Methodology 8: 19-32. doi: https://doi.org/10. 1080/1364557032000119616.

Aust, B., Nielsen, M. B. D., Grundtvig, G., Buchardt, H. L., Ferm, L., Andersen, I., Lund, T. L., Jelle, M. O. C., Andersen, M. F., Hansen, J. V., Tverborgvik, T., Helverskov, T., Bjorner, J. B., Rugulies, R., Ørbæk, P., Winzor, G., Bültmann, U., Poulsen, O. M. (2015). Implementation of the Danish return-to-work program: process evaluation of a trial in 21 Danish municipalities, Scandinavian Journal of Work, Environment \& Health 41: 529-541.

Borg, V., Nexø, M. A., Kolte, I. V., Andersen, M. F. (2010). [White paper on mental health, sickness absence and return to work], Copenhagen, The National Research Centre for the Working Environment (NFA).

Borritz, M., Rugulies, R., Christensen, K. B., Villadsen, E., Kristensen, T. S. (2006). Burnout as a predictor of self-reported sickness absence among human service workers: Prospective findings from three year follow up of the PUMA study, Occupational \& Environmental Medicine 63: 98-106. doi: https://doi.org/10.1080/14034940510032275.

Braathen, T. N., Eftedal, M., Tellnes, G., Haugli, L. (2015). Work inclusion: self-perceived change in work ability among persons in occupational rehabilitation, Vulnerable Groups and Inclusion 6: 1-25. doi: https://doi.org/10.3402/vgi.v6.26624.

Choi, B. C. K. \& Pak, A. W. P. (2006). Multidisciplinarity, interdisciplinarity and transdisciplinarity in health research, service, education and policy: 1 . Definitions, objectives, and evidence of effectiveness, Clinical \& Investigative Medicine 29: 351-364. 
Clayton, S., Barr, B., Nylen, L., Burstrom, B., Thielen, K., Didrichsen, F., Dahl, E., Whitehead, M. (2011). Effectiveness of return to work interventions for disabled people: A systematic review of government initiatives focused on changing the behaviour of employers, European Journal of Public Health 22: 434-439. doi: https://doi.org/10.1093/eurpub/ckr101.

Corbiere, M., Shen, J., Rouleau, M., Dewa, C. S. (2009). A systematic review of preventive interventions regarding mental health issues in organizations, Work: A Journal for Prevention, Assessment and Rehabilitation 33: 81-116. doi: https://doi.org/10.3233/WOR2009-0846.

Due, J. \& Madsen, J. S. (2008). The Danish model of industrial relations: Erosion or renewal? Journal of Industrial Relations 50: 513-529.

Ekberg, K. (1995). Workplace changes in successful rehabilitation, Journal of Occupational Rehabilitation 5: 253-269. doi: https://doi.org/10.1007/BF02109989.

Ekberg, K., Pransky, G. S., Besen, E., Fassier, J. B., Feuerstein, M., Munir, F., Blanck, P., and The Hopkinton Conference Working Group on Workplace Disability Prevention (2016). New business structures creating organizational opportunities and challenges for work disability prevention, Journal of Occupational Rehabilitation 26: 480-489.

Eklund, M., Wästberg, B. A., Erlandsson, L. N. (2013). Work outcomes and their predictors in the Redesigning Daily Occupations (Re DO) rehabilitation programme for women with stress-related disorders, Australian Occupational Therapy Journal 60: 85-92.

Ellström, P. E. (2008). Knowledge creation through interactive research: A learning approach. The ECER Conference, Gøteborg, Sweden.

Eriksen, H. R., Ihlebaek, C., Mikkelsen, A., Gronningsaeter, H., Sandal, G. M., Ursin, H. (2002). Improving subjective health at the worksite: A randomized controlled trial of stress management training, physical exercise and an integrated health programme, Occupational Medicine 52: 383-391.

Gahnström-Strandqvist, K., Liukko, A. Tham, K. (2003). The meaning of the working cooperative for persons with long-term mental illness: a phenomenological study, American Journal of Occupational Therapy 57: 262-272.

Gallie, K. A., Shultz, I. Z., Winter, A. (2011). Company-level interventions in mental health. In Shultz, I. Z., Rogers, E. S. (EDS.) (ed.) Work Accommodation and Retention in Mental Health, New York: Springer Science.

Geisen, T. \& Harder, H. (2011). Disability Management and Workplace Integration: International Research Findings, UK, Gower Applied Business Research.

Gensby, U. \& Husted, M. (2013). Inclusion through action: a participatory approach to return-to-work policy change processes in organisations, International Journal of Disability Management 8: 1-16.

Gensby, U., Labriola, M., Irvin, E., Amick, B. C. III., Lund, T. (2014). A classification of components of workplace disability management programs: results from a systematic review, Journal of Occupational Rehabilitation 24: 220-241.

Gensby, U., Lund, T., Kowalski, K., Saidj, M., Jørgensen, A. M. K., Filges, T., Irvin, E., Amick, B. C., Labriola, M. (2012). Workplace Disability Management Programs Promoting Return to Work: A Systematic Review. Campbell Systematic Reviews, The Campbell Collaboration.

Grossi, G. \& Santell, B. (2009). Quasi-experimental evaluation of a stress management programme for female county and municipal employees on long-term sick leave due to work-related psychological complaints, Journal of Rehabilitation Medicine 41: 632-638. doi: https://doi.org/10.2340/16501977-0379.

Gunnarson, K., Larsson, M., Schill, H. P., Josephson, M. (2014). Return to work in small workplaces, Small Enterprise Research 21: 229-237.

Habeck, R. V., Krees, M., Scully, S., Kirchner, K. (1994). Determining the significance of disability management movement for rehabilitation counselor education, Rehabilitation Education 8: 195-240. 
Habeck, R. V., Scully, S. M., Vantol, B. T, Hunt, A. H. (1998). Successful employer strategies for preventing and managing disability, Rehabilitation Counseling Bulletin 42:144-161.

Habeck, R. V. \& Hunt, A. H. (1999). Disability management perspectives: developing accommodating work environments through disability management, American Rehabilitation 25: $18-25$.

Hannes, K. \& Lockwood, C. E. (2012). Synthesizing Qualitative Research: Choosing the Right Approach, Willey-Blackwell. BMJ-Books.

Harder, G., Wagner. S., Rash, J. (2014). Mental Illness in the Workplace: Psychological Disability Management, Routledge.

Harenstam, A. (2005). Different development trends in working life and increasing occupational stress require new work environment strategies, Work: A Journal for Prevention, Assessment and Rehabilitation 24: 261-277.

Hasle, P., Hansen, N. J., Møller, N. (2004). Agreements between labour unions and employers' associations as a strategy for the prevention of repetitive strain injury, Economic and Industrial Democracy 25: 75-101.

Hasle, P., Kines, P., Andersen, L.P. (2009). Small enterprise owners' accident causation attribution and prevention, Safety Science 47: 9-19. doi: https://doi.org/10.1016/j.ssci.2007.12.005.

Hedlund, M. L. B., Wendelborg, C. (2007). Challenges in disability management of long-term sick workers, International Journal of Disability Management 2: 47-56. doi: https://doi. org/10.1375/jdmr.2.2.47.

Heijbel, B., Josephson, M., Jensen, I., Vingard, E. (2005). Employer, insurance, and health system response to long-term sick leave in the public sector: policy implications, Journal of Occupational Rehabilitation 15: 167-176.

Hjarsbech, P. U., Nielsen, M. B., Andersen, M. F., Rugulius, R., Christensen, U. (2015). Struggling at work-a qualitative study of working Danes with depressive symptoms, Disability \& Rehabilitation 37: 1674-1682. doi: https://doi.org/10.3109/09638288.2014.973970.

Holmgren, K. \& Dahlin, I. S. (2004). Women on sickness absence-views of possibilities and obstacles for returning to work. A focus group study, Disability \& Rehabilitation 26: 213-222.

Hvid, H., Bergholm, T., Gonäs, L., Juul, I., Kamp, A., Karlsson, J., Kasvio, A., Klemsdal, L., Salomon, R., Skorstad, E. (2011). Nordic working life research - continuity and renewal, Nordic Journal of Working Life Studies 1. doi: https://doi.org/10.19154/njwls.v1i1.2333.

Høgelund, J., Holm, A. (2014). Worker adaption and workplace accommodations after onset of an illness, IZA Journal of Labor Policy 3: 1-18.

Høgelund, J., Holm, A., Eplov, L. F. (2012). The effect of part-time sick leave for employees with mental disorders, Journal of Mental Health Policy and Economics 15: 157$170+197$.

ILO (2002). Managing Disability in the Workplace. ILO Code of Practice, Geneva: International Labor Organization (ILO).

Ipsen, C. \& Jensen, P. L. (2012). Organizational options for preventing work-related stress in knowledge work, International Journal of Industrial Ergonomics 42: 325-334. doi: https://doi.org/10.1016/j.ergon.2012.02.006.

Kamp, A. \& Nielsen, K. (2013). Management of Occupational Health and Safety. Nordic Lights: Work, Management and Welfare in Scandinavia, SNS förlag.

Karlson, B., Jonsson, P., Osterberg, K. (2014). Long-term stability of return to work after a workplace-oriented intervention for patients on sick leave for burnout, BMC Public Health 14: 821. doi: https://doi.org/10.1186/1471-2458-14-821.

Karlson, B., Jonsson, P., Palsson, B., Abjornsson, G., Malmberg, B., Larsson, B., Osterberg, K. (2010). Return to work after a workplace-oriented intervention for patients on sick-leave for burnout--a prospective controlled study, BMC Public Health 10: 301. doi: https://doi. org/10.1186/1471-2458-10-301. 
Karpur, A., Vanlooy, S. A., Bruyère, S. M. (2014). Employer practices for employment of people with disabilities: a literature scoping review, Rehabilitation Research, Policy and Education 28.

Krause, N. \& Lund, T. (2004). Returning to work after occupational injury. In: J. B. \& (EDS.), F. M. R. (eds.) The Psychology of Workplace Safety, Washington, DC: American Psychological Association.

Kristensen, T. S. (2008). [Is it the company the patient?]. Tidsskrift for Arbejdsliv 10: 109-113.

Kärrholm, J., Jakobsson, B., Håård, U. S., Ekholm, J., Bergroth, A., Ekholm, K. S. (2007). The views of sick-listed employees' immediate superiors on co-operation in vocational rehabilitation, Work: A Journal for Prevention, Assessment and Rehabilitation 29: 101-111.

Labriola, M. (2006). Work environment factors associated with long-term sickness absence and return to work, PhD dissertation, Department of Public Health, University of Copenhagen.

Lander, F., Friche, C., Tornemand, H., Andersen, J. H., Kirkeskov, L. (2009). Can we enhance the ability to return to work among workers with stress-related disorders? BMC Public Health 9: 372. doi: https://doi.org/10.1186/1471-2458-9-372.

Landstad, B., Vinberg, S., Ivergard, T., Gelin, G., Ekholm, J. (2001). Change in pattern of absenteeism as a result of workplace intervention for personnel support, Ergonomics 44: 63-81. doi: https://doi.org/10.1080/00140130117504.

Larsen, E. L., Labriola, M., Nielsen, C. V., Petersen, K. S. (2015). Betwixt and between: workplace perspectives on work reintegration in the eldercare sector in Denmark, Disability \& Rehabilitation 37: 1839-1848. doi: https://doi.org/10.3109/09638288.2014.982831.

Levac, D., Colquhoun, H., O'Brien, K. K. (2010). Scoping Studies: Advancing the Methodology, Implementation Science 5: 69-78.

Limborg, H. J. (2002). Flexibility as a Risk: Moving Towards a New Form of Work Environment [Den risikable fleksibilitet: På vej mod et nyt arbejdsmiljø], Copenhagen, DK, Frydenlund.

Loisel, P. \& Anema, J. R. E. (2013). Handbook of Work Disability: Prevention and Management, Canada, Springer.

Loisel, P. \& Durand, M. J. (2005). Applying Transdisciplinarity to the Complexity of Work Disability Prevention. The 2nd World Congress on Transdisciplinarity, Victoria. Brazil.

Lund, T. \& Labriola, M. (2009). Sickness absence in Denmark- Research, results, and reflections, Scandinavian Journal of Work, Environment \& Health Supplement, 5-14.

Maceachen, E., Clarke, J., Franche, R. L., Irvin, E. (2006). Systematic review of the qualitative literature on return to work after injury, Scandinavian Journal of Work, Environment and Health 32: 257-269. doi: https://doi.org/10.1007/s10926-010-9229-5.

Malachowski, C. \& Kirsh, B. (2013). Workplace antistigma initiatives: a scoping study, Psychiatric Services 64: 694-702. doi: https://doi.org/10.1176/appi.ps.201200409.

Martin, M. H., Nielsen, M. B., Madsen, I. E., Petersen, S. M., Lange, T., Rugulies, R. (2013). Effectiveness of a coordinated and tailored return-to-work intervention for sickness absence beneficiaries with mental health problems, Journal of Occupational Rehabilitation 23: 621-630. doi: https://doi.org/10.1007/s10926-013-9421-5.

Mcdowell, C. \& Fossey, E. (2015). Workplace accommodations for people with mental illness: a scoping review, Journal of Occupational Rehabilitation 25: 197-206.

Midtsundstad, T. (2008). The company as stakeholder in social policy [Virksomheten som sosialpolitisk aktør], Tidsskrift for Velferdsforskning 11: 17-33.

Murphy, L. R. \& Sauter, S. L. (2004). Work organization interventions: state of knowledge and future directions, Sozial und Präventivmedizin 49: 79-86.

Mykletun, A. \& Harvey, S. B. (2012). Prevention of mental disorders: a new era for workplace mental health, Occupational and Environmental Medicine 69: 868-869. doi: https://doi. org/10.1136/oemed-2012-100846. 
Netterstrøm, B. \& Bech, P. (2010). Effect of a multidisciplinary stress treatment programme on the return to work rate for persons with work-related stress. A non-randomized controlled study from a stress clinic, BMC Public Health 10: 658. doi: https://doi. org/10.1186/1471-2458-10-658.

Netterstrøm, B., Friebel, L., Ladegaard, Y. (2013). Effects of a multidisciplinary stress treatment programme on patient return to work rate and symptom reduction: Results from a randomised, wait-list controlled trial, Psychotherapy and Psychosomatics 82: 177-186. doi: https://doi.org/10.1159/000346369.

Nidmar, N. I. O. D. M. A. R.-. (2000). Code of Practice for Disabiltity Management, Geneve: International Labour Organization (ILO).

Nielsen, K., Randall, R., Albertsen, K. (2007). Participants' appraisals of process issues and the effects of stress management interventions, Journal of Organizational Behavior 28: 793-810.

Nielsen, M. B., Rugulius, R., Hjortkjaer, C., Bültmann, U., Christensen, U. (2012). Healing a vulnerable self: exploring return to work for women with mental health problems, Qualitative Health Research 23: 302-312. doi: https://doi.org/10.1177/1049732312468252.

Nielsen, M. L., Kristensen, T. S., Smith-Hansen, L. (2002). The Intervention Project on Absence and Well-being (IPAW): design and results from the baseline of a 5-year study, Work \& Stress 16: 191-206.

Nowotny, H. (2006). The potential of transdisciplinarity, Interdisciplines. www.interdisciplines.org/interdisciplinarity/papers/5/2/7, 5 .

Pawson, R. \& Tilley, N. (1997). Realistic Evaluation, London: Sage Publications.

Petersen, K. S., Labriola, M., Nielsen, C. V., Larsen, E. L. (2016). Work reintegration after long-term sick leave: domains of influence on co-workers' ability to be supportive, Disability \& Rehabilitation 38. doi: https://doi.org/10.3109/09638288.2015.1107772.

Peterson, U., Bergstrom, G., Samuelsson, M., Asberg, M., Nygren, A. (2008a). Reflecting peersupport groups in the prevention of stress and burnout: randomized controlled trial, Journal of Advanced Nursing 63: 506-516. doi: https://doi.org/10.1111/j.1365-2648.2008.04743.x.

Peterson, U., Demerouti, E., Bergstrom, G., Asberg, M., Nygren, A. (2008b). Work characteristics and sickness absence in burnout and nonburnout groups: a study of Swedish health care workers, International Journal of Stress Management 15: 153-172.

Petticrew, M. \& Roberts, H. (2006). Systematic Reviews in the Social Sciences, UK, Blackwell Publishing.

Pomaki, G., Franche, R. L., Murray, E., Khushrushahi, N., Lampinen, T. M. (2012). Workplace-based work disability prevention interventions for workers with common mental health conditions: a review of the literature, Journal of Occupational Rehabilitation 22: 182-195. doi: https://doi.org/10.1007/s10926-011-9338-9.

Prins, R. (2013). Sickness absence and diability: An international perspective. In: IN Loisel, P., Anema, J. R. (EDS.) (ed.) Handbook of Work Disability: Prevention and Management, Canada: Springer.

Reme, S. E., Grasdal, A. L., Lovvik, C., Lie, S. A., Overland, S. (2015). Work-focused cognitive-behavioural therapy and individual job support to increase work participation in common mental disorders: a randomised controlled multicentre trial, Occupational \& Environmental Medicine 72: 745-752. doi: https://doi.org/10.1136/oemed-2014-102700.

Saunders, S. \& Nedelec, B. (2014). What works means to people with work disability: a scoping review, Journal of Occupational Rehabilitation 24: 100-110. doi: https://doi. org/10.1007/s10926-013-9436-y.

Seing, I., Maceachen, E., Ekberg, K., Ståhl, C. (2015a). Return to work or job transition? Employer dilemmas in taking social responsibility for return to work in local workplace practice, Disability \& Rehabilitation 37: 1760-1769. doi: https://doi.org/10.3109/0963 $\underline{8288.2014 .978509 .}$. 
Seing, I., Maceachen, E., Ståhl, C., Ekberg, K. (2015b). Early-return-to-work in the context of an intensification of working life and changing employment relationships, Journal of Occupational Rehabilitation 25: 74-85. doi: https://doi.org/10.3109/09638288.2014.9 78509.

Seing, I., Ståhl, C., Nordenfelt, L., Low, P., Ekberg, K. (2012). Policy and practice of work ability: a negotiation of responsibility in organizing return to work, Journal of Occupational Rehabilitation 22: 553-564. doi: https://doi.org/10.1007/s10926-012-9371-3.

Selander, J., Tjulin, Å., Mussener, U., Ekberg, K. (2015). Contact with the workplace during long-term sickness absence and worker expectations of return to work. doi: https://doi. org/10.1017/idm.2015.3.

Stochkendahl, M. J., Myburgh, C., Young, A. E., Hartvigsen, J. (2015). Manager experiences with the return to work process in a large, publically funded, hospital setting: walking a fine line, Journal of Occupational Rehabilitation 25: 752-762. doi: https://doi. org/10.1007/s10926-015-9583-4.

Ståhl, C. (2009). Return to work collaboration: where are the employers? [Samverkan kring återgång i arbete: var är arbetsgivarna?], Social Medicisnk Tidsskrift 86: 265-274.

Ståhl, C. (2010). In Cooperation We Trust: Inter-organizational Cooporation in Return to Work and Labour Market Reintegration, Linköping University.

Ståhl, C., Edvardsson, Stiwne E. (2014). Narratives of sick leave, return to work and job mobility for people with common mental disorders in Sweden, Journal of Occupational Rehabilitation 24: 543-554. doi: https://doi.org/10.1007/s10926-013-9480-7.

Svensson, L., Ellström, P. E., Brulin, G. (2009). Organizing interactive research in a triple helix context: A mode III perspective. The HSSO9 Conference, Luleå. Sweden.

Tjulin, Å., Maceachen, E., Ekberg, K. (2010). Exploring workplace actors experiences of the social organization of return-to-work, Journal of Occupational Rehabilitation 20: 311-321. doi: https://doi.org/10.1007/s10926-009-9209-9.

Tjulin, A., Maceachen, E., Ekberg, K. (2011a). Exploring the meaning of early contact in return-to-work from workplace actors' perspective, Disability \& Rehabilitation 33: 137145. doi: https://doi.org/10.3109/09638288.2010.489630.

Tjulin, Å., Maceachen, E., Stiwne, E. E., Ekberg, K. (2011b). The social interaction of return to work explored from co-workers experiences, Disability \& Rehabilitation 33: 1979_ 1989. doi: https://doi.org/10.3109/09638288.2011.553708.

Tjulin, Å., Mussener, U., Selander, J., Ekberg, K. (2015). Learning experiences in return to work among workplace actors, International Journal of Disability Management. doi: https://doi.org/10.1017/idm.2015.1.

Tveito, T. H. \& Eriksen, H. R. (2009). Integrated health programme: a workplace randomized controlled trial, Journal of Advanced Nursing 65: 110-119. doi: https://doi.org/10.1111/ j.1365-2648.2008.04846.x.

Van Eerd, D. \& Saunders, R. (2017). Integrated knowledge transfer and exchange: An organizational approach for stakeholder engagement and communications, Scholarly and Research Communication 8: 1-18.

Vinberg, S. \& Landstad, B. J. (2014). Workplace-based prevention and rehabilitation programs in Swedish public human service organisations, International Journal of Disability Management. doi: https://doi.org/10.1017/idm.2014.1.

Vingaard, E. (2015). Mental Health Problems, Working Life and Sickness Absence: A Review of the Literature [Psykisk ohälsa, arbetsliv och sjukfrånvaro: En kunnskapsoversigt], Stockholm: The Research Council for Health, Working Life and Welfare (FORTE).

WHO (2006). Mental Health Policies and Programmes in the Workplace. In: PACKAGE, M. H. P. A. S. G. (ed.), Geneva, Switzerland: World Health Organisation.

Wästberg, B. A., Erlandsson, L. K., Eklund, M. (2016). Women's perceived work environment after stress-related rehabilitation: experiences from the ReDO project, Disability \& Rehabilitation 38: 528-534. doi: https://doi.org/10.3109/09638288.2015.1046567. 
Wåhlin, C., Ekberg, K., Persson, J., Bernfort, L., Öberg, B. (2012). Association between clinical and work-related interventions and return-to-work for patients with musculoskeletal or mental disorders, Journal of Rehabilitation Medicine 44: 355-362. doi: https://doi. org/10.2340/16501977-0951.

Wåhlin, C., Ekberg, K., Persson, J., Bernfort, L., Öberg, B. (2013). Evaluation of self-reported work ability and usefulness of interventions among sick-listed patients, Journal of Occupational Rehabilitation 23: 32-43. doi: https://doi.org/10.1007/s10926-012-9376-y. 


\section{Appendix I: Venn diagram of search concepts and associated} key terms

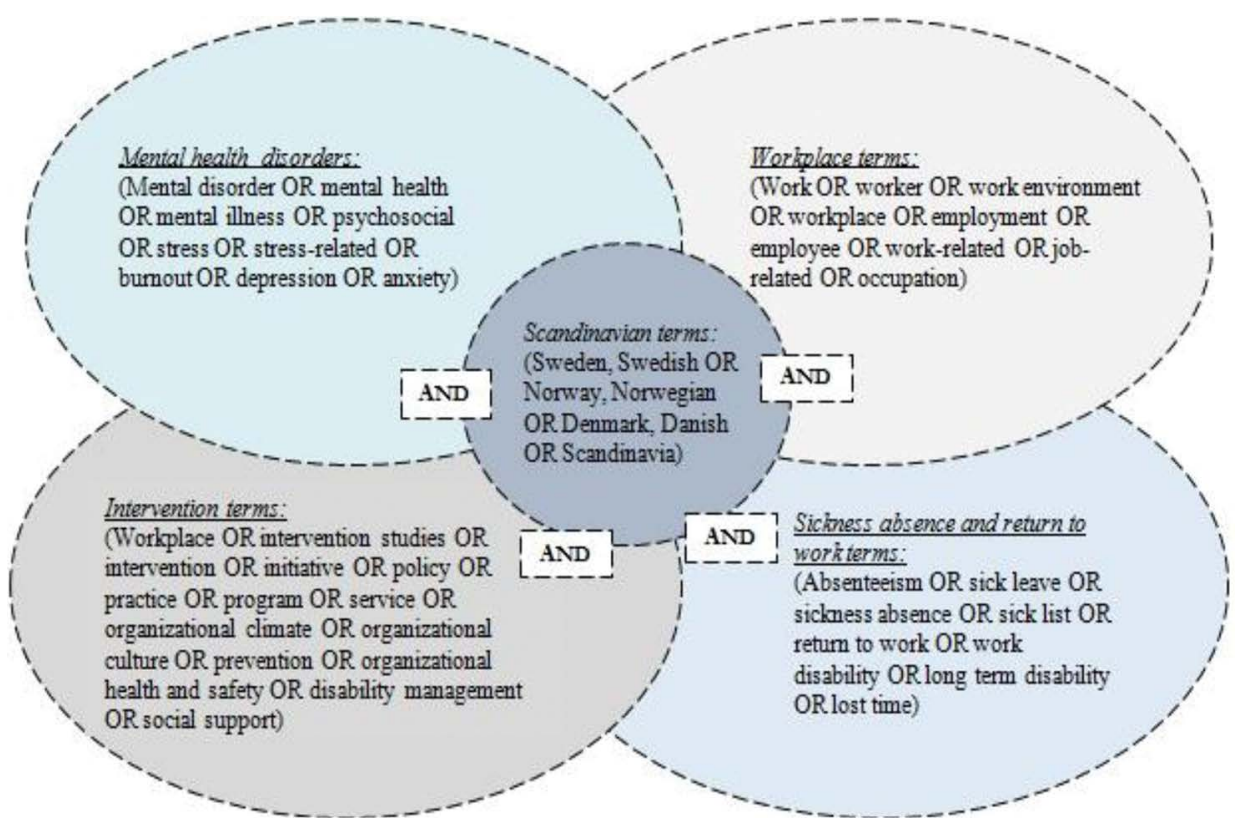




\section{Appendix 2: Inclusion criteria for study selection}

\section{Identifying relevant studies (Title and abstract screening)}

- Is the study a peer reviewed research study? Yes/No?

- Is the study about sickness absence and/or RTW? Yes/No?

- Is the study targeting the magnitude of CMD disorders? (i.e. stress-related disorder/ psychological distress, anxiety, burnout, and/or depression) Yes/No?

Selection of relevant studies (Full text screening)

- Is the study using data sources from a Scandinavian setting (DK, NO, SVE)? Yes/No?

- Does the study include participants being in an employment relationship (i.e., fulltime, part time, or reduced hours), and/or consider one or more workplace stakeholders in the development, implementation, or evaluation of preventive activities? Yes/No?

- Does the study address psychosocial working environment research about one or multiple workplace initiative (s) to prevent sickness absence due to CMD? Yes/No?

- Does the study address DM research about organizational policy and/or work practice implementation, or change (s) promoting RTW? Yes/No?

Study exclusion reasons

- Not peer-reviewed research study (i.e., conference presentation, or editorial)

- Not on sickness absence/RTW

- Not Scandinavian context/setting

- Severe psychiatric conditions (i.e., bipolar disorder, psychosis, and schizophrenia).

- Unemployed persons taking part in vocational rehabilitation, and/or people in early retirement preparing to move into or finding a job.

- Stand-alone clinical or social insurance interventions without direct involvement of the workplace, and/or a clear workplace initiative or work practice change to prevent CMD-related work disability. 